\title{
The occurrence of nitrogen-enhanced metal-poor stars: implications for the initial mass function in the early Galactic halo ${ }^{\star}$
}

\author{
O. R. Pols ${ }^{1,2}$, R. G. Izzard ${ }^{3,4}$, R. J. Stancliffe ${ }^{5,6}$, and E. Glebbeek ${ }^{1,7}$
}

\author{
1 Department of Astrophysics/IMAPP, Radboud University Nijmegen, PO Box 9010, 6500 GL Nijmegen, The Netherlands \\ e-mail: o.pols@astro.ru.nl \\ 2 Sterrenkundig Instituut Utrecht, PO Box 80000, 3508 TA Utrecht, The Netherlands \\ 3 Argelander-Institut für Astronomie, Auf dem Hügel 71, 53121 Bonn, Germany \\ ${ }^{4}$ Institut d'Astronomie et d'Astrophysique, Université Libre de Bruxelles, Boulevard du Triomphe, 1050 Brussels, Belgium \\ 5 School of Mathematical Sciences, PO Box 28M, Monash University, 3800 Victoria, Australia \\ ${ }^{6}$ Research School of Astronomy \& Astrophysics, Mount Stromlo Observatory, Cotter Road, Weston Creek ACT 2611, Australia \\ 7 Department of Physics and Astronomy, McMaster University, Hamilton, Ontario, L8S 4M1, Canada
}

Received 14 May 2012 / Accepted 19 September 2012

\begin{abstract}
Most carbon-enhanced metal-poor (CEMP) stars are thought to result from past mass transfer of He-burning material from an asymptotic giant branch (AGB) star to a low-mass companion star, which we now observe as a CEMP star. Because AGB stars of intermediate mass efficiently cycle carbon into nitrogen in their envelopes, the same evolution scenario predicts the existence of a population of nitrogen-enhanced metal-poor (NEMP) stars, with $[\mathrm{N} / \mathrm{Fe}]>1$ and $[\mathrm{N} / \mathrm{C}]>0.5$. Such NEMP stars are rare, although their occurrence depends on metallicity: they appear to be more common at $[\mathrm{Fe} / \mathrm{H}]<-2.8$ by about a factor of 10 compared to less metal-poor stars. We analyse the observed sample of metal-poor stars with measurements of both carbon and nitrogen to derive firm constraints on the occurrence of NEMP stars as a function of metallicity. We compare these constraints to binary population synthesis calculations in which we vary the initial distributions of mass, mass ratio and binary orbital periods. We show that the observed paucity of NEMP stars at $[\mathrm{Fe} / \mathrm{H}]>-2.8$ does not allow for large modifications in the initial mass function, as have been suggested in the literature to account for the high frequency of CEMP stars. The situation at lower metallicity is less clear, and we do not currently have stellar models to perform this comparison for $[\mathrm{Fe} / \mathrm{H}]<-2.8$. However, unless intermediate-mass AGB stars behave very differently at such low metallicity, the observed NEMP frequency at $[\mathrm{Fe} / \mathrm{H}]<-2.8$ appears incompatible with the top-heavy forms of the initial mass function suggested in the literature.
\end{abstract}

Key words. stars: carbon - binaries: close - stars: chemically peculiar - Galaxy: halo - Galaxy: stellar content nuclear reactions, nucleosynthesis, abundances

\section{Introduction}

Carbon-enhanced metal-poor (CEMP) stars make up a large proportion of the most metal-poor stars in the Galactic halo. Estimates of the fraction of very metal-poor stars (with $[\mathrm{Fe} / \mathrm{H}]<$ -2) having carbon enhancements $[\mathrm{C} / \mathrm{Fe}]>1.0$ range between $10 \%$ and 25\% (Frebel et al. 2006; Lucatello et al. 2006; Carollo et al. 2012). Based on their abundance patterns different subgroups are identified among the CEMP stars (Beers \& Christlieb 2005; Masseron et al. 2010). By far the most numerous group are also enriched in $s$-process elements, which make up about $80 \%$ of CEMP stars (Aoki et al. 2007). We refer to this group as CEMP-s stars, noting that a certain fraction of these are also enriched in $r$-process elements (these have been designated as CEMP-rs stars in the literature). Radial velocity monitoring suggests that probably all CEMP-s stars are binaries (Lucatello et al. 2005b). A widely accepted formation scenario for this group involves pollution by mass transfer in a binary system from a more massive asymptotic giant branch (AGB) companion, which has since become a white dwarf. Of the CEMP

* Appendix $\mathrm{A}$ is available in electronic form at http: //WwW . aanda.org stars without strong $s$-process enhancements, one star is found to be strongly enriched in $r$-process elements, the remainder having no or only weak overabundances of heavy elements. The latter group are known as CEMP-no stars. The origin of these stars is less clear; there is no strong evidence for a large binary fraction among the CEMP-no stars. They appear to be more common at the lowest metallicities. Although they may have followed a similar evolution path as the CEMP-s stars, it is also possible that they have an entirely different origin (e.g. Meynet et al. 2010).

Within the mass transfer scenario, the large proportion of CEMP-s stars requires the existence of a sufficient number of binary systems with primary components that have undergone AGB nucleosynthesis. Several authors (Abia et al. 2001; Lucatello et al. 2005a; Komiya et al. 2007) have therefore argued that a different initial mass function (IMF), weighted towards intermediate-mass stars, is needed at low metallicity. Lucatello et al. (2005a) find that the standard IMF for the Galactic disk (Miller \& Scalo 1979) contains too few AGB progenitors in the mass range $1.2-6 M_{\odot}$, assumed to be responsible for carbon and $s$-element production, to explain the observed fraction of CEMP-s stars. They propose an IMF with a mild shift towards intermediate-mass stars is required. Komiya et al. (2007) 
present a semi-analytical model for binary population synthesis, based on AGB models in which low-mass stars $\left(0.8-1.5 M_{\odot}\right)$ at $[\mathrm{Fe} / \mathrm{H}]<-2.5$ produce carbon and $s$-elements by dual shell flashes (see Sect. 5 for details). While this increases the parameter space for making CEMP-s stars considerably, it is barely sufficient to produce a CEMP-s fraction of more than $10 \%$ with a Galactic-disk IMF. They further propose that the CEMP-no stars are produced in binary systems with AGB primaries of 3.5-6 $M_{\odot}$. From an assumed CEMP-no/CEMP-s ratio of at least $1 / 3$, consistent with observations available to them, they find consistency with both the CEMP-s fraction (10-25\%) and the CEMP-no/CEMP-s ratio for a top-heavy IMF, with a median mass of $10 M_{\odot}$. Such proposed changes to the IMF would have profound consequences, since the IMF affects such diverse quantities as the mass-to-light ratio of galaxies, the overall production of heavy elements and galactic chemical evolution.

However, the model calculations on which these conclusions are based still contain many uncertainties regarding the evolution and nucleosynthesis of low-metallicity AGB stars, the efficiency of mass transfer, and the evolution of the surface abundances of the CEMP stars themselves. A different approach to the problem of ubiquity of CEMP stars was taken by Izzard et al. (2009). They performed detailed binary population synthesis calculations to explore whether the observed CEMP-s fraction at $[\mathrm{Fe} / \mathrm{H}] \approx-2.3$ can be explained within the boundaries of the model uncertainties, while assuming a standard solarneighbourhood IMF. Their results confirmed that with current detailed AGB models, in which dredge-up of carbon occurs only for masses larger than $1.2 M_{\odot}$, the large CEMP fraction cannot be explained. However, they also found that efficient dredgeup in lower-mass AGB stars $\left(0.8-1.2 M_{\odot}\right)$ can substantially increase the expected CEMP fraction to within the observed range. Therefore the question of whether CEMP stars provide evidence for a modified IMF at low metallicity remains open.

Apart from carbon, substantial enhancements of nitrogen with respect to iron are common among CEMP stars, typically with $[\mathrm{N} / \mathrm{C}]<0$. Detailed AGB nucleosynthesis models of low initial mass $\left(<3-5 M_{\odot}\right.$, depending on metallicity) make carbon through the dredge-up of helium-burning products, but produce very little nitrogen. On the other hand, AGB stars of higher mass are predicted to convert the dredged-up carbon into nitrogen by $\mathrm{CN}$ cycling at the bottom of the convective envelope (hot bottom burning, HBB). The surface abundances of these more massive $\mathrm{AGB}$ stars approach the $\mathrm{CN}$ equilibrium ratio of $[\mathrm{N} / \mathrm{C}] \approx 2$. Detailed evolution models of AGB stars (Karakas \& Lattanzio 2007) indicate that, while at solar metallicity HBB sets in at $\gtrsim 5 M_{\odot}$, significantly lower masses are needed at low metallicity. At $[\mathrm{Fe} / \mathrm{H}]=-2.3$ the lower mass limit is found to be $\approx 3 M_{\odot}$ (Karakas 2010). By the same mass-transfer scenario that forms CEMP-s stars, one may thus expect a population of so-called nitrogen-enhanced metal-poor (NEMP) stars, with enhanced nitrogen abundances and $[\mathrm{N} / \mathrm{C}]>0.5$. Note that we thus expect NEMP stars to result from a similar AGB mass range that Komiya et al. (2007) suggested to produce CEMP-no stars (based on models in which no HBB occurs). Although a few examples of NEMP stars are known, mostly at $[\mathrm{Fe} / \mathrm{H}]<-2.9$, they appear to be very rare (Johnson et al. 2007).

In this paper we show that the number of NEMP stars sets an additional constraint on possible changes to the IMF at low metallicity. In Sect. 2 we discuss the observational evidence regarding NEMP stars. Our population synthesis models are described in Sect. 3 and the results are presented in Sect. 4. We discuss our results in Sect. 5 and in Sect. 6 we give our conclusions.

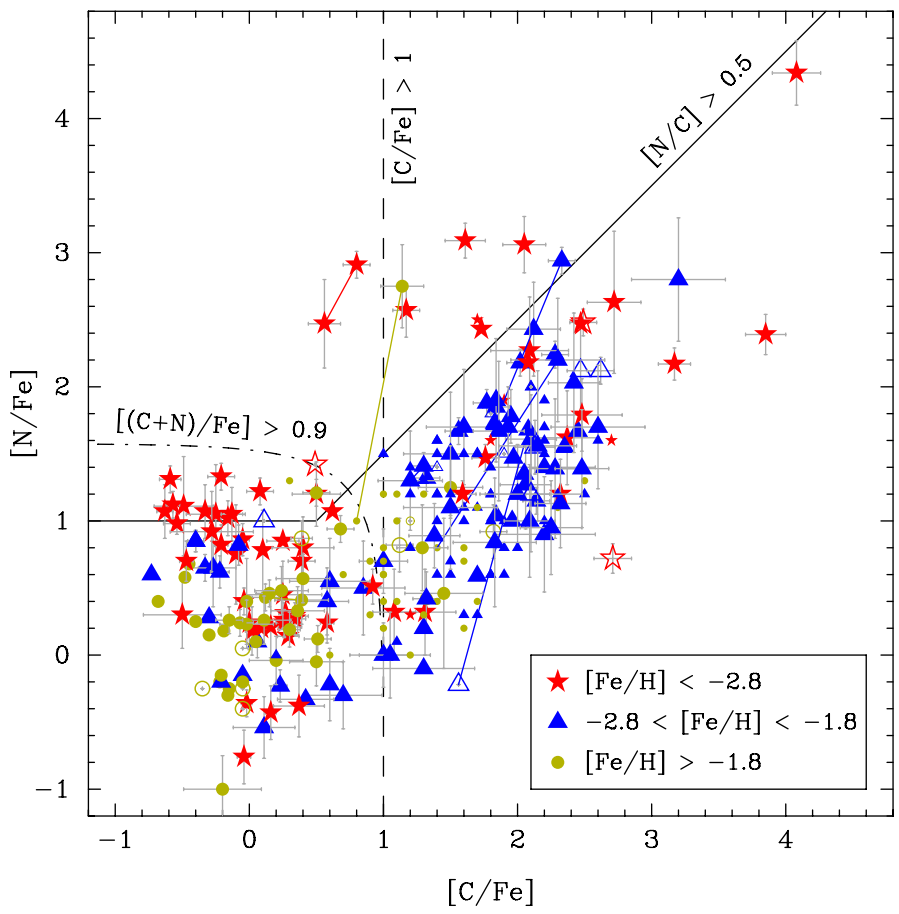

Fig. 1. Distribution of $[\mathrm{N} / \mathrm{Fe}]$ versus $[\mathrm{C} / \mathrm{Fe}]$ for metal-poor stars with observed carbon and nitrogen abundances. Star-shaped (red) symbols are stars with $[\mathrm{Fe} / \mathrm{H}]<-2.8$, triangles (dark blue) have $-2.8 \leq$ $[\mathrm{Fe} / \mathrm{H}] \leq-1.8$ and circles (yellow) have $[\mathrm{Fe} / \mathrm{H}]>-1.8$. Giants (stars with $\log g \leq 4$ ) are shown as filled symbols while higher-gravity stars have open symbols. Large symbols represent data from Masseron et al. (2010) and the SAGA database; data from Lucatello et al. (2006) is shown with smaller symbols. Multiple data for the same star are connected with solid line segments. The dashed and solid lines indicate the criteria adopted for CEMP stars and NEMP stars, and the dash-dotted line is the alternative criterion $[(\mathrm{C}+\mathrm{N}) / \mathrm{Fe}]>0.9$ (see text).

\section{Nitrogen-enhanced metal-poor stars}

Johnson et al. (2007) used the same theoretical considerations as we gave above to argue for the expected existence of a substantial population of NEMP stars, which they defined as stars with $[\mathrm{N} / \mathrm{Fe}]>0.5$ and $[\mathrm{N} / \mathrm{C}]>0.5$. They set out to search for NEMP stars by determining nitrogen and carbon abundances for a sample of 21 mildly carbon-enhanced stars. They found no NEMP stars but confirmed the known trend that $[\mathrm{N} / \mathrm{C}]$ values of CEMP stars tend to be intermediate between what is expected from AGB stars with masses above and below the hot bottom burning limit. While their study showed that NEMP stars are rare, and their sample has the advantage of being homogeneous, it is too small to derive firm constraints on the frequency of NEMP stars. We therefore performed a literature search to obtain a more complete overview of the situation.

Characterising a star as NEMP requires measurements of both the $\mathrm{C}$ and $\mathrm{N}$ abundances, which are only available for a limited number of halo stars. In Fig. 1 we show the distribution of $[\mathrm{N} / \mathrm{Fe}]$ versus $[\mathrm{C} / \mathrm{Fe}]$ of 259 metal-poor stars with measurements of both carbon and nitrogen. Data for 176 stars have been collected in the SAGA database (Suda et al. 2008, 2011) and by Masseron et al. (2010). These datasets largely overlap but Masseron et al. list a few stars not included in SAGA. Many stars have multiple measurements of both $\mathrm{C}$ and $\mathrm{N}$ that are broadly consistent within the observational errors, in which case we generally take the data from the most recent publication as listed by Masseron et al. (2010). For a few stars with clearly discrepant 
O.R. Pols et al.: Nitrogen-enhanced metal-poor stars

Table 1. Nitrogen-enhanced metal-poor stars, and other possible remnants of intermediate-mass binaries (last two entries).

\begin{tabular}{|c|c|c|c|c|c|c|c|c|c|}
\hline Star & {$[\mathrm{Fe} / \mathrm{H}]$} & $\log g$ & {$[\mathrm{C} / \mathrm{Fe}]$} & {$[\mathrm{N} / \mathrm{Fe}]$} & ${ }^{12} \mathrm{C} /{ }^{13} \mathrm{C}$ & {$[\mathrm{Na} / \mathrm{Fe}]$} & {$[\mathrm{Mg} / \mathrm{Fe}]$} & {$[\mathrm{Ba} / \mathrm{Fe}]$} & Reference \\
\hline \multirow[t]{2}{*}{ CS22949-037 } & -3.86 & 1.7 & +1.07 & +2.11 & & +1.45 & +1.50 & -0.59 & $\mathrm{C} 08$ \\
\hline & -3.97 & 1.5 & +1.17 & +2.57 & 3 & +2.09 & +1.58 & -0.58 & D02 \\
\hline \multirow[t]{2}{*}{ CS22960-053 } & -3.14 & 2.1 & +2.05 & +3.06 & & & +0.65 & +0.86 & A07 \\
\hline & -3.08 & 2.4 & +1.15 & +1.15 & & & & & J07 \\
\hline CS29528-041 & -3.30 & 4.0 & +1.61 & +3.09 & & +1.20 & +0.45 & +0.97 & S06 \\
\hline \multirow{2}{*}{ CS30314-067 } & -2.85 & 0.7 & +0.50 & +1.20 & 5 & -0.08 & +0.42 & -0.57 & A02 \\
\hline & -2.76 & 1.0 & +0.25 & +0.50 & & & & & J07 \\
\hline \multirow[t]{2}{*}{ CS30322-023 } & -3.39 & -0.3 & +0.80 & +2.91 & 4 & +1.29 & +0.80 & +0.52 & M06, M10 \\
\hline & -3.25 & 1.0 & +0.56 & +2.47 & & +1.04 & +0.54 & +0.59 & A07 \\
\hline HD 25329 & -1.8 & 4.7 & +0.11 & +1.00 & $>40$ & +0.24 & +0.59 & +0.11 & G00, G03, F00 \\
\hline HD 206983 & -0.99 & 0.6 & +0.50 & +1.21 & 5 & & +0.26 & +0.92 & M10 \\
\hline \multirow[t]{2}{*}{ HE0400-2030 } & -1.73 & 3.5 & +1.14 & +2.75 & & +0.71 & +0.62 & +1.64 & A07 \\
\hline & -1.7 & 2.5 & +0.8 & +1.0 & & & & & L06 \\
\hline HE1031-0020 & -2.93 & 2.2 & +1.73 & +2.43 & & & +0.53 & +1.28 & C06 \\
\hline HE1337+0012 ${ }^{a}$ & -3.20 & 4.4 & +0.49 & +1.42 & & -1.13 & +0.41 & -0.25 & A06 \\
\hline \multirow[t]{2}{*}{ HE1410+0213 } & -2.52 & 2.0 & +2.33 & +2.94 & 3 & & +0.33 & +0.05 & M10 \\
\hline & -2.23 & 3.5 & +1.83 & +1.73 & & & +0.21 & +0.14 & $\mathrm{C} 06$ \\
\hline HE1413-1954 & -2.9 & 4.0 & +1.7 & +2.5 & & & & & L06 \\
\hline HE2150-0825 & -1.3 & 3.8 & +0.3 & +1.3 & & & & & L06 \\
\hline HE2253-4217 & -2.4 & 1.6 & +1.0 & +1.5 & & & & & L06 \\
\hline CS29528-028 & -2.86 & 4.0 & +2.77 & $<+3.58$ & & +2.68 & +1.69 & +3.27 & A07 \\
\hline SDSS1707+58 & -2.52 & 4.2 & +2.1 & & & +2.71 & +1.13 & +3.40 & A08 \\
\hline
\end{tabular}

Notes. ${ }^{(a)}$ Also known as G64-12.

References. A02: Aoki et al. (2002), A06: Aoki et al. (2006), A07: Aoki et al. (2007), A08: Aoki et al. (2008), C06: Cohen et al. (2006), C08: Cohen et al. (2008), D02: Depagne et al. (2002), F00: Fulbright (2000), G00: Gratton et al. (2000), G03: Gratton et al. (2003), J07: Johnson et al. (2007), L06: Lucatello et al. (2006), M06: Masseron et al. (2006), M10: Masseron et al. (2010), S06: Sivarani et al. (2006).

measurements, we show two data points connected by a line segment. Added to this selection are 87 stars, mostly CEMP, with $\mathrm{C}$ and $\mathrm{N}$ abundances determined by Lucatello et al. (2006), which were not included in the SAGA database. We note that the Johnson et al. (2007) data are included in SAGA.

Following Izzard et al. (2009, hereinafter Paper I), we designate as CEMP stars those with $[\mathrm{C} / \mathrm{Fe}] \geq 1.0$ and as NEMP stars those with

$$
[\mathrm{N} / \mathrm{Fe}] \geq 1.0 \quad \text { and } \quad[\mathrm{N} / \mathrm{C}] \geq 0.5 \text {. }
$$

Note that these definitions partly overlap; stars that satisfy both criteria (i.e. stars with $[\mathrm{C} / \mathrm{Fe}] \geq 1.0$ and $[\mathrm{N} / \mathrm{C}] \geq 0.5$ ) are designated as CNEMP stars. The adopted CEMP and NEMP criteria are shown as dashed and solid lines in Fig. 1.

Our NEMP criterion (1) is somewhat more restrictive on the nitrogen abundance than the definition of Johnson et al. (2007). Application of this criterion to our selection of data yields 24 NEMP star candidates (see also Table 2 of Paper $\mathrm{I}^{1}$ ). Ten of these stars, however, have $[\mathrm{C} / \mathrm{Fe}] \lesssim 0.0$ and $[\mathrm{N} / \mathrm{Fe}]$ values between 1.0 and 1.3. All these stars have $[\mathrm{Fe} / \mathrm{H}]<-2.8$ and are on the upper part of the red giant branch (RGB): they belong to the sample of "mixed stars" of Spite et al. (2005, 2006). Their nitrogen enhancements are very likely the result of $\mathrm{CN}$ cycling and "extra mixing" on the RGB itself, and not the result of mass accretion of nitrogen-rich material. Indeed, their combined $\mathrm{C}+$ $\mathrm{N}$ abundances are only mildly super-solar, $[(\mathrm{C}+\mathrm{N}) / \mathrm{Fe}] \lesssim 0.7$. Our aim of eliminating such stars from our selection by the criterion $[\mathrm{N} / \mathrm{Fe}] \geq 1.0$ was obviously not completely successful.

\footnotetext{
1 We found 17 stars in Paper I. The difference is due partly to the addition of new data and partly to our use in Paper I of the average observed abundance, while here we use data from a single source.
}

An alternative, and perhaps better, criterion to distinguish stars that have had significant accretion of He-burning products (either carbon or nitrogen) can be based on the $\mathrm{C}+\mathrm{N}$ abundance, because $\mathrm{CN}$-cycling during $\mathrm{HBB}$ conserves the total number of $\mathrm{C}+\mathrm{N}$ nuclei. If carbon is enhanced by a factor greater than 10 (corresponding to our CEMP criterion $[\mathrm{C} / \mathrm{Fe}] \geq$ 1.0) and subsequently cycled partly into nitrogen, we expect $[(\mathrm{C}+\mathrm{N}) / \mathrm{Fe}] \geq 0.9$, assuming Grevesse et al. (1996) solarscaled initial abundances. This criterion is shown as the dashdotted line in Fig. 1. It effectively eliminates stars that are N-rich owing to internal mixing processes on the RGB. In this paper, however, we prefer to use our criterion (1) because it is consistent with Paper I and our model calculations, but we will subject NEMP stars with $[(\mathrm{C}+\mathrm{N}) / \mathrm{Fe}]<0.9$ to critical examination.

If we eliminate the 10 "mixed" RGB stars we are left with 14 NEMP candidates, for which we list several key properties in Table 1. However, not all of these stars necessarily qualify as "genuine" NEMP stars, i.e., stars that are likely to have been polluted by nitrogen-rich material, possibly from an intermediate-mass AGB star. Other clues can be obtained from the ${ }^{12} \mathrm{C} /{ }^{13} \mathrm{C}$ ratio, which is expected to be close to its $\mathrm{CN}$ equilibrium value of 3-4 for material that has gone through $\mathrm{HBB}$, and from measurements of $\mathrm{Na}$ and $\mathrm{Mg}$. In an AGB star undergoing $\mathrm{HBB}, \mathrm{Na}$ is produced by the $\mathrm{NeNa}$ chain from the abundant ${ }^{22} \mathrm{Ne}$ brought to the surface by third dredge-up, along with $\mathrm{Mg}$ if the ${ }^{22} \mathrm{Ne}(\alpha, \mathrm{n})^{25} \mathrm{Mg}$ source is active. Therefore $[\mathrm{Na} / \mathrm{Fe}]$ and $[\mathrm{Mg} / \mathrm{Fe}]$ values greater than zero are expected in material lost by an intermediate-mass AGB star (e.g. see Lugaro et al. 2012), although we note that Na production by AGB stars is uncertain owing to the large uncertainty in the ${ }^{22} \mathrm{Ne}(\mathrm{p}, \gamma)^{23} \mathrm{Na}$ reaction rate (Izzard et al. 2007). Clearly, not all NEMP candidates in Table 1 for which ${ }^{12} \mathrm{C} /{ }^{13} \mathrm{C}, \mathrm{Na}$ and/or $\mathrm{Mg}$ are measured show the expected behaviour. In particular, HD 25329 is an unevolved 
A\&A 547, A76 (2012)

Table 2. Incidence of CEMP, NEMP and CNEMP stars among stars with carbon and nitrogen measurements.

\begin{tabular}{lccccccc}
\hline \hline Selection & CEMP & NEMP & CNEMP & $f_{\text {NEMP }}$ & $f_{\max }(90 \% / 99 \% / 99.9 \%)$ & $f_{\text {CNEMP }}$ & $f_{\max }(90 \% / 99 \% / 99.9 \%)$ \\
\hline all & 156 & 14 & 8 & 0.086 & & 0.051 & \\
all, $\log g \leq 4$ & 142 & 12 & 8 & 0.082 & $0.122 / 0.156 / 0.185$ & 0.056 & $0.092 / 0.123 / 0.149$ \\
\hline$[\mathrm{Fe} / \mathrm{H}]<-2.8$ & 26 & 8 & 5 & 0.276 & & 0.192 & \\
{$[\mathrm{Fe} / \mathrm{H}]<-2.8, \log g \leq 4$} & 24 & 7 & 5 & 0.269 & $0.411 / 0.514 / 0.588$ & 0.208 & $0.352 / 0.459 / 0.539$ \\
$-2.8 \leq[\mathrm{Fe} / \mathrm{H}] \leq-1.8$ & 101 & 3 & 2 & 0.029 & & 0.020 & \\
$-2.8 \leq[\mathrm{Fe} / \mathrm{H}] \leq-1.8, \log g \leq 4$ & 92 & 2 & 2 & 0.022 & $0.057 / 0.088 / 0.116$ & 0.022 & $0.057 / 0.088 / 0.116$ \\
{$[\mathrm{Fe} / \mathrm{H}]>-1.8$} & 29 & 3 & 1 & 0.097 & & 0.034 & \\
{$[\mathrm{Fe} / \mathrm{H}]>-1.8, \log g \leq 4$} & 26 & 3 & 1 & 0.107 & $0.223 / 0.316 / 0.390$ & 0.038 & $0.142 / 0.229 / 0.304$ \\
\hline$[\mathrm{Fe} / \mathrm{H}] \geq-2.8$ & 130 & 6 & 3 & 0.045 & & 0.023 & \\
{$[\mathrm{Fe} / \mathrm{H}] \geq-2.8, \log g \leq 4$} & 118 & 5 & 3 & 0.042 & $0.076 / 0.106 / 0.131$ & 0.025 & $0.056 / 0.083 / 0.106$ \\
\hline
\end{tabular}

Notes. The columns labelled $f_{\max }$ give the $90 \%, 99 \%$ and $99.9 \%$ upper limits to $f_{\mathrm{NEMP}}$ and $f_{\mathrm{CNEMP}}$ for the selections with $\log g \leq 4$.

main-sequence star with $[\mathrm{N} / \mathrm{Fe}]=1.0$ and $[\mathrm{C} / \mathrm{Fe}]=0.1$, and thus a modest $[(\mathrm{C}+\mathrm{N}) / \mathrm{Fe}]=0.5$. Its ${ }^{12} \mathrm{C} /{ }^{13} \mathrm{C}$ ratio is at least 40 which is inconsistent with accretion of material that has undergone HBB. Although the origin of its high $\mathrm{N}$ abundance is unclear, we should probably exclude this star from the sample of genuine NEMP stars. We comment in more detail on the stars in Table 1 in Appendix A.

Figure 1 demonstrates the paucity of NEMP stars, in particular among the more metal-rich part of the population. We focus in particular on the metallicity range $-2.8 \leq[\mathrm{Fe} / \mathrm{H}] \leq-1.8$ because these stars can be best compared to our models at $[\mathrm{Fe} / \mathrm{H}]=-2.3$. Within this metallicity range (triangles) there are only three candidate NEMP stars, one of which (HD 25329) we can most likely exclude. The other two are also doubtful cases: HE1410+0213 has an alternative measurement that gives $[\mathrm{N} / \mathrm{C}]<0$, and HE2253-4217 is exactly on the borderline of our criterion with $[\mathrm{N} / \mathrm{C}]=0.5$ and $[\mathrm{C} / \mathrm{Fe}]=1.0$. Thus, there may be no NEMP stars in the $[\mathrm{Fe} / \mathrm{H}]$ range that we can directly compare with our models at $[\mathrm{Fe} / \mathrm{H}]=-2.3$. In contrast, NEMP stars appear to be much more common among the low-metallicity population $([\mathrm{Fe} / \mathrm{H}]<-2.8$, star symbols $)$. However, we are dealing with low-numbers statistics so we need to take care in drawing such conclusions. We therefore perform a more careful analysis of the statistics of NEMP stars.

\subsection{Statistics of CEMP and NEMP stars}

It is difficult to derive an overall fraction of NEMP stars from the observed samples. The latest release of the SAGA database of metal-poor stars (dated Oct. 31, 2010; Suda et al. 2011) contains 656 stars with measurements of the carbon abundance, of which 110 (about 17\%) classify as CEMP stars. For 148 stars the nitrogen abundance has also been determined. Out of these, 68 have $[\mathrm{C} / \mathrm{Fe}]>1$, amounting to a CEMP fraction of $46 \%$ among stars with both $\mathrm{C}$ and $\mathrm{N}$ measurements. Apparently there is a strong bias towards selecting CEMP stars as targets for nitrogen abundance determinations. The Masseron et al. (2010) and Lucatello et al. (2006) samples of stars with $\mathrm{C}$ and $\mathrm{N}$ measurements are even more strongly biased towards CEMP stars. One reason for this bias may be that in most studies nitrogen abundances are determined from the $\mathrm{CN}$ band, which requires the presence of a substantial amount of carbon.

On the other hand, among stars for which both $\mathrm{C}$ and $\mathrm{N}$ abundances are measured there is no reason to suspect a strong bias towards certain $[\mathrm{N} / \mathrm{C}]$ values. We can thus expect that the number ratio of NEMP to CEMP stars among these stars is a fairly unbiased observable. We designate as $f_{\mathrm{NEMP}}$ the fraction of NEMP stars among stars that are either CEMP or NEMP (i.e. $f_{\mathrm{NEMP}}=N_{\mathrm{NEMP}} /\left[N_{\mathrm{CEMP}}+N_{\mathrm{NEMP}}-N_{\mathrm{CNEMP}}\right]$, which avoids counting CNEMP stars twice) such that $f_{\text {NEMP }} \leq 1$ by definition. The common use of $\mathrm{CN}$ as a nitrogen abundance indicator may bias the observed sample of NEMP stars towards C-rich stars in such a way that non-C-rich NEMP stars are underrepresented in the observed sample. We therefore also consider the fraction of CNEMP stars among CEMP stars, which we denote as $f_{\text {CNEMP }}$ and which should be unaffected by such a bias. Another advantage of using the (C)NEMP/CEMP ratio as a constraint on our model predictions is that it is independent of various model uncertainties, most notably the binary fraction. We therefore attempt to derive $f_{\mathrm{NEMP}}$ and $f_{\mathrm{CNEMP}}$ from the 162 stars that are classified as either CEMP or NEMP from the combined SAGA-Masseron-Lucatello sample as shown in Fig. 1. From the 14 NEMP stars and 8 CNEMP stars we have found in this sample (Table 1 ) we thus deduce a nominal $f_{\mathrm{NEMP}}=0.086$ and $f_{\text {CNEMP }}=0.051$ in the entire sample.

However, this number deserves closer scrutiny for two reasons. Firstly, Fig. 1 and Table 1 demonstrate that most NEMP stars have rather low $[\mathrm{Fe} / \mathrm{H}]$ and the NEMP/CEMP ratio appears to depend on metallicity. This dependence becomes clear when we split up the sample into different $[\mathrm{Fe} / \mathrm{H}]$ ranges, see Table 2. Among stars with $[\mathrm{Fe} / \mathrm{H}]<-2.8$ both $f_{\text {NEMP }}$ and $f_{\text {CNEMP }}$ are about 10 times higher than in the range $-2.8 \leq[\mathrm{Fe} / \mathrm{H}] \leq-1.8$.

Secondly, the numbers quoted in Table 2 include stars of uncertain NEMP status, so we can at best derive upper limits to the NEMP/CEMP fraction from the observations. Since we are dealing with very small numbers, especially when we consider subsamples in different metallicity ranges, we cannot simply take the nominal fractions in Table 2. Instead we should ask the question: given a true fraction $f$ of NEMP stars among CEMP stars, what is the probability of finding at most $N$ NEMP stars in an observed sample of $N_{\text {tot }}$ CEMP stars? Assuming a purely stochastic process, this probability is $P(\leq N) \equiv \sum_{n=0}^{N} P_{\mathrm{b}}\left(n, N_{\mathrm{tot}}, f\right)$, where $P_{\mathrm{b}}\left(n, N_{\text {tot }}, f\right)$ is the binomial probability of drawing $n$ NEMP stars out of a sample of $N_{\text {tot }}$ given a true fraction $f$. Since $P(\leq N)$ is a decreasing function of $f$, if we find $P(\leq N)$ to be, say, $10 \%$ for a certain value of $f$, we can exclude NEMP/CEMP fractions larger than $f$ with 90 per cent confidence. In this way we can compute upper limits to $f$ for certain confidence limits, i.e. $90 \%$, $99 \%$ and $99.9 \%$. These upper limits are given in Table 2 for the different subsamples and for both $f_{\text {NEMP }}$ and $f_{\text {CNEMP }}$.

As in Izzard et al. (2009) we limit our analysis to stars with $\log g \leq 4$, i.e. subgiants and giants. This facilitates the 
comparison with our model calculations and does not introduce significant biases in the numbers we derive, as can be verified in Table 2. We further limit the selection to stars with $-2.8 \leq$ $[\mathrm{Fe} / \mathrm{H}] \leq-1.8$, which is the relevant metallicity range for comparison to our models at $[\mathrm{Fe} / \mathrm{H}]=-2.3$. The SAGA database contains 287 stars with $\mathrm{C}$ measurements in this range, out of which 59 are CEMP stars. This amounts to a CEMP fraction ${ }^{2}$ of $20.6 \%$. Adding the additional CEMP stars from Masseron et al. (2010) and Lucatello et al. (2006) yields 92 CEMP stars and 2 NEMP stars, both of which are also (marginally) CNEMP. Despite a nominal $f_{\text {NEMP }}$ of 0.022 in this range, we cannot exclude real fractions up to 0.088 with more than $99 \%$ confidence.

The NEMP/CEMP ratio appears to be larger at both lower and higher metallicity. The difference at lower metallicity is likely to be real, since at least 5 out of the 7 NEMP stars at $[\mathrm{Fe} / \mathrm{H}]<-2.8$ appear to be genuine. We can compute the probability that we observe at least 5 NEMP stars among 26 CEMP+NEMP stars given a real fraction $f$ in a similar way, and find we can exclude $f<0.097$ with $90 \%$ confidence and $f<0.052$ with $99 \%$ confidence. On the other hand, the status of the 3 NEMP stars at $[\mathrm{Fe} / \mathrm{H}]>-1.8$ is less certain and the high nominal NEMP/CEMP ratio could just be due to chance and small-number statistics. We can take these stars into account by combining them with the $-2.8 \leq[\mathrm{Fe} / \mathrm{H}] \leq-1.8$ sample, see the lower line of Table 2 . We then find upper limits to $f_{\text {NEMP }}$ that are only slightly larger than from the $-2.8 \leq[\mathrm{Fe} / \mathrm{H}] \leq 1.8$ sample by itself, while the limits to $f_{\text {CNEMP }}$ are even slightly lower.

We conclude by noting that the SAGA database is by no means a statistically complete sample. Bright giants are clearly overrepresented with respect to fainter giants and turnoff stars, compared to what is expected from the relative lifetimes of these phases (Izzard et al. 2009). However, neither the observed sample nor our model results show a strong dependence of the CEMP fraction on evolution state (represented e.g. by the effective gravity). Therefore the results we describe below are probably not greatly affected by this selection effect.

\section{Population synthesis models}

We have simulated populations of metal-poor halo stars in binary systems using the rapid synthetic binary nucleosynthesis code of Izzard et al. (2004, 2006). The algorithm and the assumptions made in these simulations are discussed extensively in Paper I. We adopt a metallicity $Z=10^{-4}([\mathrm{Fe} / \mathrm{H}]=-2.3)$ and select stars with ages between 10 and $13.7 \mathrm{Gyr}$ (roughly corresponding to the age of the halo) and $\log g \leq 4.0$. Our synthetic populations contain only binaries (see Sect. 5 for a discussion of the binary fraction). However, the predicted NEMP/CEMP fraction is independent of the binary fraction, assuming both CEMP and NEMP stars are formed through binary mass transfer.

We consider models A, G and H from Paper I, to which we refer for details. Model A is our default model, where we parameterize AGB evolution, including the efficiency of third dredgeup (TDU), according to the detailed models of Karakas et al. (2002). This yields C-rich AGB stars and thus CEMP progenitors for initial primary masses $M_{1}>1.2 M_{\odot}$. Hot bottom burning produces $\mathrm{N}$-rich AGB stars for $M_{1}>2.7 M_{\odot}$ in these models. In Model $\mathrm{G}$ we assume much more efficient TDU in low-mass AGB stars by reducing the minimum core mass and envelope

\footnotetext{
2 In Izzard et al. (2009) we found a larger CEMP fraction, 31\%, from a similar selection on $[\mathrm{Fe} / \mathrm{H}]$ and $\log g$. However, the dataset used in that paper combined the SAGA database with a selection of the data from Lucatello et al. (2006) which is strongly biased towards CEMP stars.
}

mass required for dredge-up, as described in Paper I. This results in almost all stars with initial masses $0.85-2.7 M_{\odot}$ becoming Crich AGB stars and thus potential CEMP progenitors. On the other hand, more massive stars - and thus NEMP progenitors are not affected by this choice. We note that more efficient TDU is also required to match the carbon-star luminosity functions in the Magellanic Clouds (Izzard et al. 2004). Models A and G both assume efficient thermohaline mixing of the accreted matter throughout the stellar envelope of the secondary. Model $\mathrm{H}$ is similar to Model $\mathrm{G}$ but we do not allow thermohaline mixing, such that accreted material remains at the surface of the secondary with unchanged abundances until first dredge-up occurs.

In this study, in addition to varying the main assumptions about the input physics, we also vary the initial binary parameter distributions as detailed below. We have no direct information about these distributions in the halo at low metallicity, and must necessarily make some rather crude assumptions. Models 1-4 are alternative representations of the binary population in the solar neighbourhood, with which we test the sensitivity of our results to uncertainties in these distributions.

1. Default model, as in Paper I: the initial primary masses $M_{1}$ are distributed according to the solar neighbourhood IMF as derived by Kroupa et al. (1993), the initial separations are drawn from a flat distribution in $\log a$ (with $a$ between 3 and $10^{5} R_{\odot}$ ) and the initial mass ratios from a flat distribution in $q=M_{2} / M_{1}$. The IMF and mass-ratio distribution for this model are shown as solid lines in Figs. 2b and c. A flat distribution in $\log a$ provides the best description of the binary population of young OB associations (Kouwenhoven et al. 2007), and thus represents intermediate-mass binary populations in the Galaxy (with $M_{1} \gtrsim 3 M_{\odot}$ ).

2. Primary masses and mass ratios are distributed as in Model 1, but orbital periods are drawn from the distributions derived by Duquennoy \& Mayor (1991, hereafter DM91) for the local population of $\mathrm{G}$ dwarfs (with $M_{1} \sim 1 M_{\odot}$ ), i.e. a lognormal period distribution with a broad peak at 170 years.

3. As model 2, but here also the mass ratios are drawn from the distribution proposed by DM91, i.e. a Gaussian mass-ratio distribution with a broad peak at $q=0.23$. This is shown as the dashed line in Fig. 2c.

4. Primary masses are taken from an alternative IMF for the solar neighbourhood by Miller \& Scalo (1979), described by a log-normal distribution,

$$
\frac{\mathrm{d} N}{\operatorname{dlog} M_{1}} \propto \exp \left[-\frac{\left(\log M_{1}-\log M_{\mathrm{med}}\right)^{2}}{2 \sigma_{M}{ }^{2}}\right],
$$

with a median mass of $M_{\text {med }}=0.1 M_{\odot}$ and a width $\sigma_{M}=$ 0.67 , while separations and mass ratios are distributed as in Model 1.

Models 5 and 6 test the effect of two alternative forms of the IMF that have been suggested in the literature.

5. As Model 4, but with a modified log-normal IMF (Eq. (2)) with $M_{\text {med }}=0.79 M_{\odot}$ and $\sigma_{M}=0.5$, as proposed by Lucatello et al. (2005a). This IMF is shown as a dashed line in Fig. $2 b$.

6. As Model 4 using a log-normal IMF with a much larger median mass of $10 M_{\odot}$ and a narrower width, $\sigma_{M}=0.33$, as proposed by Komiya et al. (2007).

We also test the effect of varying the IMF parameters in Eq. (2), $M_{\text {med }}$ and $\sigma_{M}$, in a continuous manner between $0.1 M_{\odot}$ and $20 M_{\odot}$ and between 0.1 and 1.0 , respectively. 


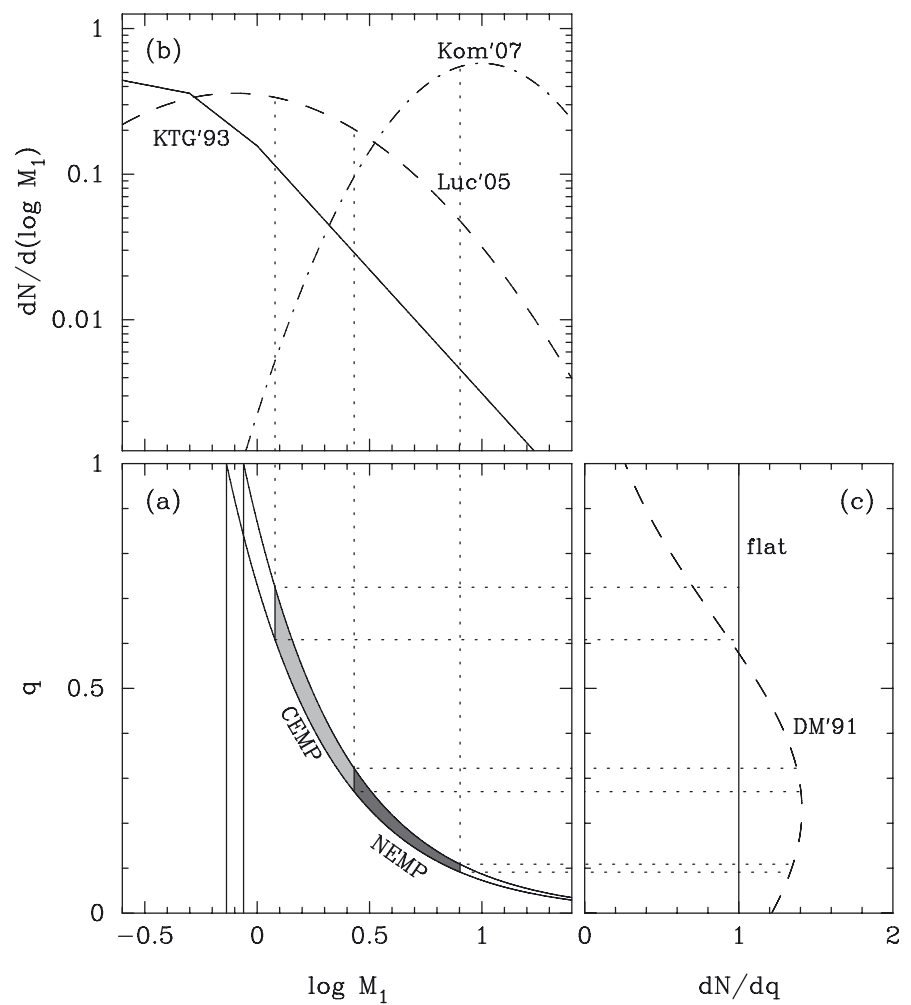

Fig. 2. a) Schematic regions in the parameter space of initial primary mass $M_{1}$ and mass ratio $q$ for the progenitors of EMP stars $(M \approx$ $0.8 M_{\odot}$, between the solid lines) and of CEMP and NEMP stars (light and dark shading, respectively); see text for details. b) Three examples of the initial primary mass function (Kroupa et al. 1993; Lucatello et al. 2005a; Komiya et al. 2007). c) Comparison of two initial mass-ratio distributions.

\section{Results}

Figure 2a shows in a schematic way where the progenitors of CEMP and NEMP stars are located in the parameter space of initial primary mass $M_{1}$ and initial mass ratio $q$. The constraints on age and gravity mean that we select stars with masses in a narrow interval around $0.8 M_{\odot}$. These stars are either primary components in binaries (indicated by the vertical lines in Fig. 2a) or the original secondaries in binaries where the more massive primary has evolved into a compact remnant. The latter have $M_{2}=q M_{1} \approx 0.8 M_{\odot}$, which defines a small range of mass ratios $\Delta q \propto 1 / M_{1}$ for each $M_{1}$ (shown by the curved lines) ${ }^{3}$. The CEMP and NEMP progenitors lie in the grey-shaded regions in Fig. 2a, where the mass limits shown correspond to Model A. Only binaries with orbital periods in a certain range evolve into CEMP or NEMP stars; this is not shown, but our models indicate that this period range is not very sensitive to $M_{1}$. Another simplification in Fig. 2 is that we have ignored accretion: in reality the initial mass ratios of CEMP and NEMP progenitors are somewhat smaller because the secondaries accrete up to $0.1 M_{\odot}$ of primary material.

This figure illustrates several effects that are visible in the results we obtain. First, both the CEMP fraction among stars with $M \approx 0.8 M_{\odot}$ and the NEMP/CEMP ratio depend on the shape of the IMF, both increasing as the median mass of stars formed increases. Second, since $\Delta q \propto 1 / M_{1}$, the fraction of binary systems

\footnotetext{
${ }^{3}$ Note that in Fig. 2 the indicated width $\Delta M$ of the mass interval around $0.8 M_{\odot}$ is arbitrary and only for illustration purposes; this cancels out when calculating stellar number ratios as long as $\Delta M \ll M$.
}

Table 3. Predicted number fractions of CEMP, NEMP, and CNEMP stars among halo stars at $[\mathrm{Fe} / \mathrm{H}] \sim-2.3$ and $\log g<4.0$.

\begin{tabular}{lrllll}
\hline \hline Model & CEMP & NEMP & CNEMP & $f_{\text {NEMP }}$ & $f_{\text {CNEMP }}$ \\
\hline A1 & $2.33 \%$ & $0.27 \%$ & $0.10 \%$ & 0.107 & 0.041 \\
A2 & $2.75 \%$ & $0.34 \%$ & $0.11 \%$ & 0.114 & 0.041 \\
A3 & $3.50 \%$ & $0.54 \%$ & $0.18 \%$ & 0.139 & 0.051 \\
A4 & $3.18 \%$ & $0.48 \%$ & $0.17 \%$ & 0.137 & 0.051 \\
A5 & $4.87 \%$ & $1.04 \%$ & $0.36 \%$ & 0.188 & 0.074 \\
A6 & $13.3 \%$ & $19.7 \%$ & $5.4 \%$ & 0.714 & 0.406 \\
\hline G1 & $9.5 \%$ & $0.27 \%$ & $0.10 \%$ & 0.027 & 0.011 \\
G2 & $11.1 \%$ & $0.34 \%$ & $0.12 \%$ & 0.030 & 0.011 \\
G3 & $8.5 \%$ & $0.53 \%$ & $0.19 \%$ & 0.060 & 0.023 \\
G4 & $10.6 \%$ & $0.48 \%$ & $0.18 \%$ & 0.044 & 0.017 \\
G5 & $12.7 \%$ & $1.04 \%$ & $0.39 \%$ & 0.078 & 0.031 \\
G6 & $15.6 \%$ & $19.7 \%$ & $5.8 \%$ & 0.667 & 0.372 \\
\hline H1 & $15.8 \%$ & $0.43 \%$ & $0.30 \%$ & 0.027 & 0.019 \\
H2 & $18.7 \%$ & $0.55 \%$ & $0.38 \%$ & 0.029 & 0.021 \\
H3 & $15.0 \%$ & $0.87 \%$ & $0.60 \%$ & 0.057 & 0.040 \\
H4 & $17.9 \%$ & $0.77 \%$ & $0.53 \%$ & 0.042 & 0.030 \\
H5 & $22.1 \%$ & $1.68 \%$ & $1.16 \%$ & 0.074 & 0.052 \\
H6 & $43.5 \%$ & $35.8 \%$ & $22.8 \%$ & 0.634 & 0.525 \\
\hline
\end{tabular}

Notes. The last two columns give the fraction $f_{\text {NEMP }}$ of NEMP stars among stars that are either CEMP or NEMP and the fraction $f_{\text {CNEMP }}$ of CNEMP stars among CEMP stars.

at a certain $M_{1}$ that evolve into a CEMP or NEMP star decreases with primary mass. For a flat mass-ratio distribution this fraction is, to first order, inversely proportional to $M_{1}$. This effect results in a smaller NEMP/CEMP ratio than one would expect on the basis of the IMF alone. Third, NEMP progenitor systems have smaller initial mass ratios $(0.1 \lesssim q \lesssim 0.3)$ than CEMP progenitors $(0.3 \lesssim q \lesssim 0.7$ for Model A). Therefore the NEMP/CEMP ratio is sensitive to the initial mass-ratio distribution. On the other hand, because the orbital periods of CEMP and NEMP progenitors are similar, the NEMP/CEMP ratio is not sensitive to the initial period distribution.

In Table 3 we give the number fractions of CEMP, NEMP and CNEMP stars resulting from our models, for various assumptions regarding the physical ingredients (models A, G and $\mathrm{H}$ ) and the initial distributions of binary parameters $(1-6)$. We also compute the (C)NEMP/CEMP fractions $f_{\mathrm{NEMP}}$ and $f_{\text {CNEMP }}$ from these models for comparison with the observational constraints derived in Sect. 2.1. Since our models are for $[\mathrm{Fe} / \mathrm{H}]=-2.3$, this comparison should strictly be made only with the subsample having $-2.8 \leq[\mathrm{Fe} / \mathrm{H}] \leq-1.8$. A substantial fraction of NEMP stars in our models are CNEMP, because even though intermediate-mass AGB stars convert most dredged-up carbon into nitrogen by $\mathrm{HBB}$, these stars still produce large amounts of carbon. This material can be further diluted by thermohaline mixing in the secondary and therefore in model sets $A$ and $\mathrm{G}$ a smaller proportion of NEMP stars are CNEMP than in model set $\mathrm{H}$, in which no such dilution occurs until first dredge-up.

The results for models with default binary parameter distributions (A1, G1 and H1) are discussed extensively in Paper I. Our default model (A1) fails to account for the large observed CEMP frequency, while Model G1 yields an increase of the CEMP fraction to almost $10 \%$, substantially closer to the observed value. This corresponds to decreasing the lower primary mass limit of CEMP progenitors in Fig. 2 to about $0.85 M_{\odot}$. Inhibiting thermohaline mixing increases the CEMP frequency to more than $15 \%$ (Model H1). In all these default models the 
small overall NEMP fraction $(<0.5 \%)$ is consistent with the observations, and Models G1 and H1 with enhanced dredge-up can comfortably reproduce the observed small $f_{\text {NEMP }}$. Based on the considerations in Sect. 2.1, Model A1 can be rejected with more than $99 \%$ confidence on the basis of its $f_{\mathrm{NEMP}}$ value - but this is entirely due to the small CEMP fraction in this model. If we consider only CNEMP stars, the constraints are weaker and also Model A1 (with $f_{\text {CNEMP }}=0.041$ ) cannot be excluded with more than $90 \%$ confidence.

\subsection{Variations in parameter distributions of the local binary population}

Distributions 2 and 3 give some insight into the dependence of our results on uncertainties in the local (solar-neighbourhood) binary parameter distributions. Model A2 shows a modest increase in both the number of CEMP stars and NEMP stars compared to Model A1, because the peak in the Duquennoy \& Mayor (1991) period distribution coincides with the period range in which mass transfer is most effective. This is partly compensated by the wider range of periods in the DM91 distribution. Similarly modest effects are seen when considering Models G2 and $\mathrm{H} 2$. $f_{\mathrm{NEMP}}$ and $f_{\mathrm{CNEMP}}$ are hardly affected by this choice.

Adopting also the DM91 mass-ratio distribution in Model A3 gives a further increase in the CEMP and NEMP fractions, by factors of 1.5 and 2 compared to Model A1, as this distribution favours the initial mass ratios with which most CEMP stars and all NEMP stars are born in model set A (see Fig. 2c). A similar increase in the number of NEMP stars is seen in model sets $\mathrm{G}$ and $\mathrm{H}$. On the other hand, in Model G3 the number of CEMP stars is somewhat smaller than in Model G1 because most CEMP stars now come from binaries with primary masses $0.85-1.2 M_{\odot}$ and $q>0.7$, which is disfavoured by the DM91 mass-ratio distribution (Fig. 2c). The same applies to Model H3. In Models G3 and H3 $f_{\mathrm{NEMP}}$ and $f_{\mathrm{CNEMP}}$ increase by a factor of 2 compared to the default distribution. While less likely to be correct, these models cannot be excluded with more than $90 \%$ confidence (Table 2).

Adoption of the Miller \& Scalo (1979) IMF (Models A4, G4 and H4) gives somewhat higher CEMP and NEMP fractions than the Kroupa et al. (1993) IMF, but the effect is modest: less than factors of 1.4 and 1.8 for the CEMP and NEMP stars, respectively. We conclude that uncertainties in the distributions of the solar-neighbourhood binary populations affect the CEMP and NEMP fraction and their ratio by at most a factor of 2 .

\subsection{Modified forms of the initial mass function}

Our results for distributions 5 and 6 show the effect of a modified IMF. Model A5 assumes the IMF suggested by Lucatello et al. (2005a) as being required to reproduce the large CEMP fraction. The fraction of CEMP stars increases by a factor of 2 compared to the KTG93 IMF (Model A1), as this IMF favours intermediate-mass stars over low-mass stars (Fig. 2b). However, the CEMP fraction still falls short of the observed value. Our CEMP fraction is smaller than Lucatello et al. (2005a) predict because our ranges of initial primary mass and orbital period that produce CEMP stars are more restrictive. With enhanced third dredge-up the increase in the number of CEMP stars is only a modest factor of 1.3 (Model G5) to 1.4 (Model H5). Models A5-H5 also show an increased (C)NEMP fraction, by a factor of 4 over models A1-H1, respectively, which is the direct result of a larger weight of intermediate-mass stars in model A

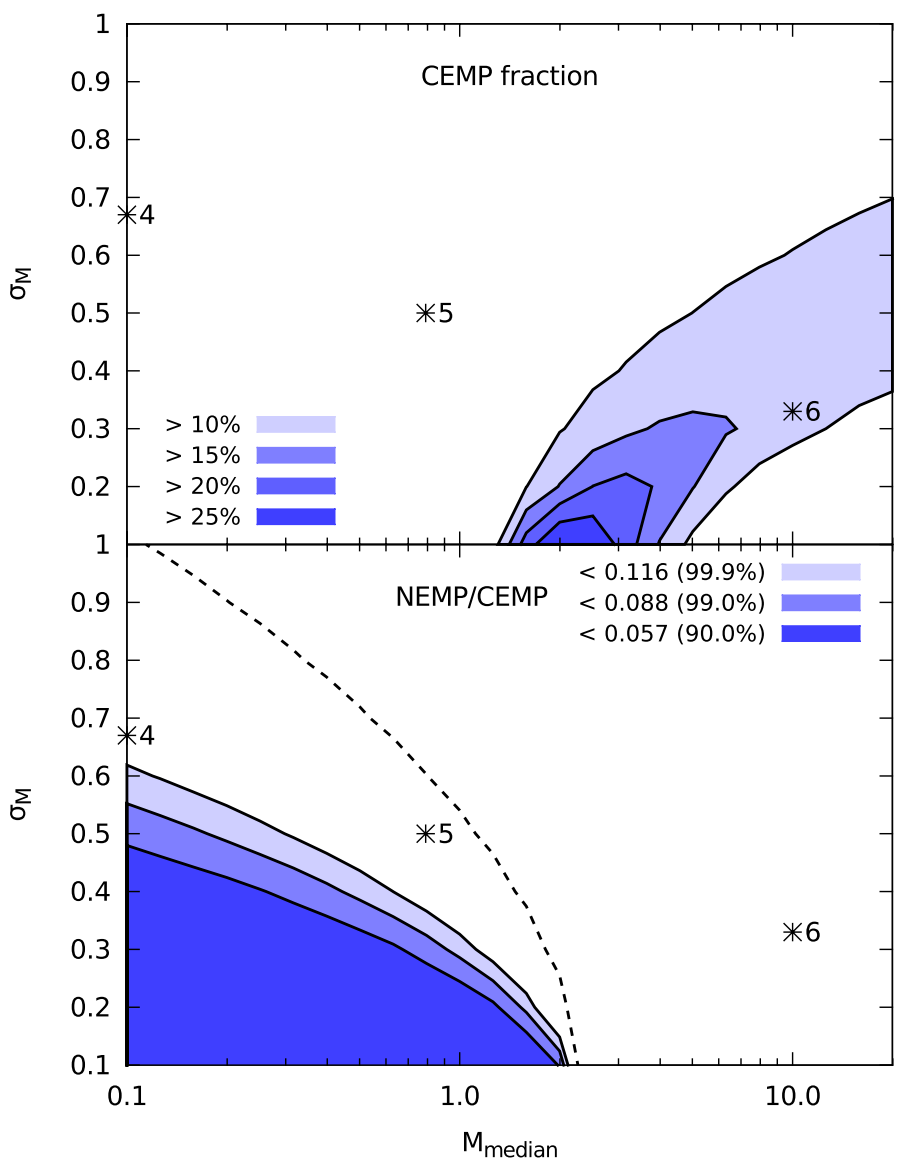

Fig. 3. Dependence of the CEMP fraction (upper panel) and the fraction $f_{\text {NEMP }}$ of NEMP stars among all CEMP+NEMP stars (lower panel) for our default model A, as a function of the median mass $M_{\text {med }}$ and dispersion $\sigma_{M}$ for a log-normal IMF. Contours in the upper panel correspond to CEMP fractions between $10 \%$ and $25 \%$, which spans the range of observed values. The solid contours in the lower panel correspond to the $90 \%, 99 \%$ and $99.9 \%$ confidence upper limits on $f_{\mathrm{NEMP}}$ in the range $-2.8 \leq[\mathrm{Fe} / \mathrm{H}] \leq-1.8$ and $\log g \leq 4$, while the dashed line shows the $99 \%$ upper limit on $f_{\text {CNEMP }}$ (see Table 2 ). The star symbols labelled 4 , 5 and 6 indicate the IMFs of Miller \& Scalo (1979), Lucatello et al. (2005a) and Komiya et al. (2007).

this IMF. Models G5 and H5 have $f_{\text {NEMP }} \sim 0.07-0.08$ and $f_{\text {CNEMP }} \sim 0.03-0.05$, which is still (marginally) compatible with the observed limits and cannot be excluded with high confidence. We note that model $\mathrm{H} 5$, which combines efficient dredgeup, inhibited thermohaline mixing and a modest change to the IMF, is the only model that comfortably reproduces a CEMP fraction of about $20 \%$ while remaining consistent with the small observed number of NEMP stars at $[\mathrm{Fe} / \mathrm{H}]>-2.8$.

The effects noted above are much more extreme when we assume the IMF suggested by Komiya et al. (2007), which has a median mass of $10 M_{\odot}$. This IMF gives rise to a substantial CEMP fraction, even with default input physics (Model A6), although it remains smaller than $20 \%$ even in Model G6: the effect of enhanced dredge-up is only modest in combination with this IMF. However, the main effect of such a top-heavy IMF is an enormous increase in the number of NEMP stars. One would expect about $20 \%$ of metal-poor stars to be NEMP and more than 5\% to be CNEMP according to Models A6 and G6, and these numbers are even higher (36\% NEMP and 22\% CNEMP) 
model G

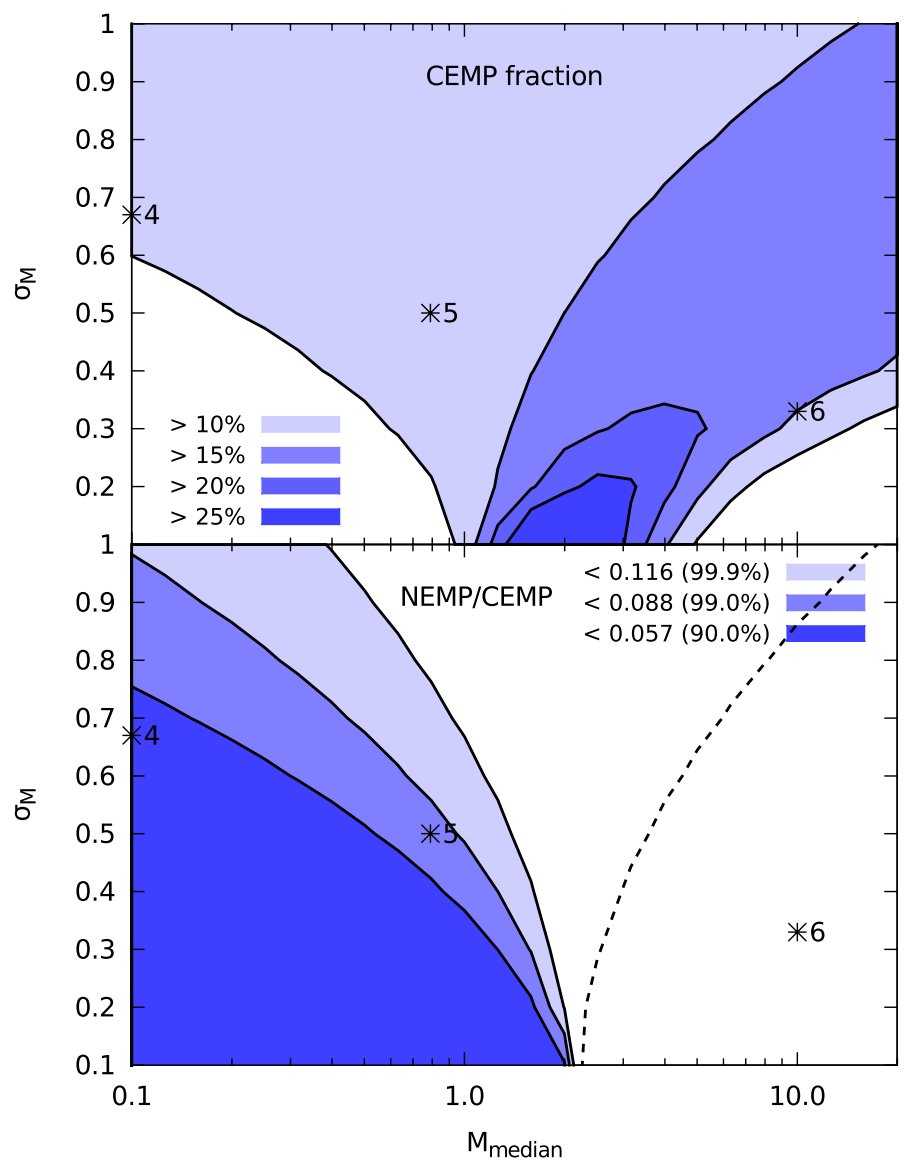

Fig. 4. Same as Fig. 3 for model G, with enhanced dredge-up in lowmass AGB stars.

in Model H6. In these models $f_{\mathrm{NEMP}}$ and $f_{\mathrm{CNEMP}}$ are strongly incompatible with the observed limits, at least for $[\mathrm{Fe} / \mathrm{H}]>-2.8$.

On the other hand, given the much larger apparent $f_{\mathrm{NEMP}}$ among stars with $[\mathrm{Fe} / \mathrm{H}]<-2.8$ one might more plausibly consider a top-heavy IMF at these low metallicities. Although we cannot really compare our models to this low-metallicity sample, we might assume that the results in Table 3 are not sensitive to the metallicity and also apply to $[\mathrm{Fe} / \mathrm{H}]<-2.8$. If this were the case, then using the analysis of Sect. 2.1 we find the probability of finding at most 7 NEMP stars among the sample of 26 CEMP and NEMP stars to be only $3 \times 10^{-6}, 4 \times 10^{-5}$ and $2 \times 10^{-4}$ for Models A6, G6 and H6, respectively. Considering $f_{\text {CNEMP }}$, the probability of finding 5 CNEMP stars among the 24 CEMP stars would be $3.5 \%, 7 \%$ and $0.16 \%$ for Models A6, G6 and H6, respectively. This crude analysis suggests that the data at $[\mathrm{Fe} / \mathrm{H}]<-2.8$ do not support a Komiya et al. (2007) topheavy IMF, unless many non-C-rich NEMP stars have remained undiscovered.

We investigate the constraints on the IMF further in Figs. 3-5, where we convolve our model calculations for Models $\mathrm{A}, \mathrm{G}$ and $\mathrm{H}$, respectively, with a log-normal IMF of the form (2) using the median mass and width of the distribution as free parameters. The top panels show contours of the CEMP fraction while the bottom panels show contours of $f_{\mathrm{NEMP}}$, both as a function of $M_{\text {med }}$ and $\sigma_{M}$. The contours in the lower panels correspond to the $90 \%, 99 \%$ and $99.9 \%$ confidence limits on $f_{\text {NEMP }}$ and the $99 \%$ limit on $f_{\text {CNEMP }}$ (dashed line) for $-2.8<[\mathrm{Fe} / \mathrm{H}]<-1.8$, see Table 2 . This is analogous to the

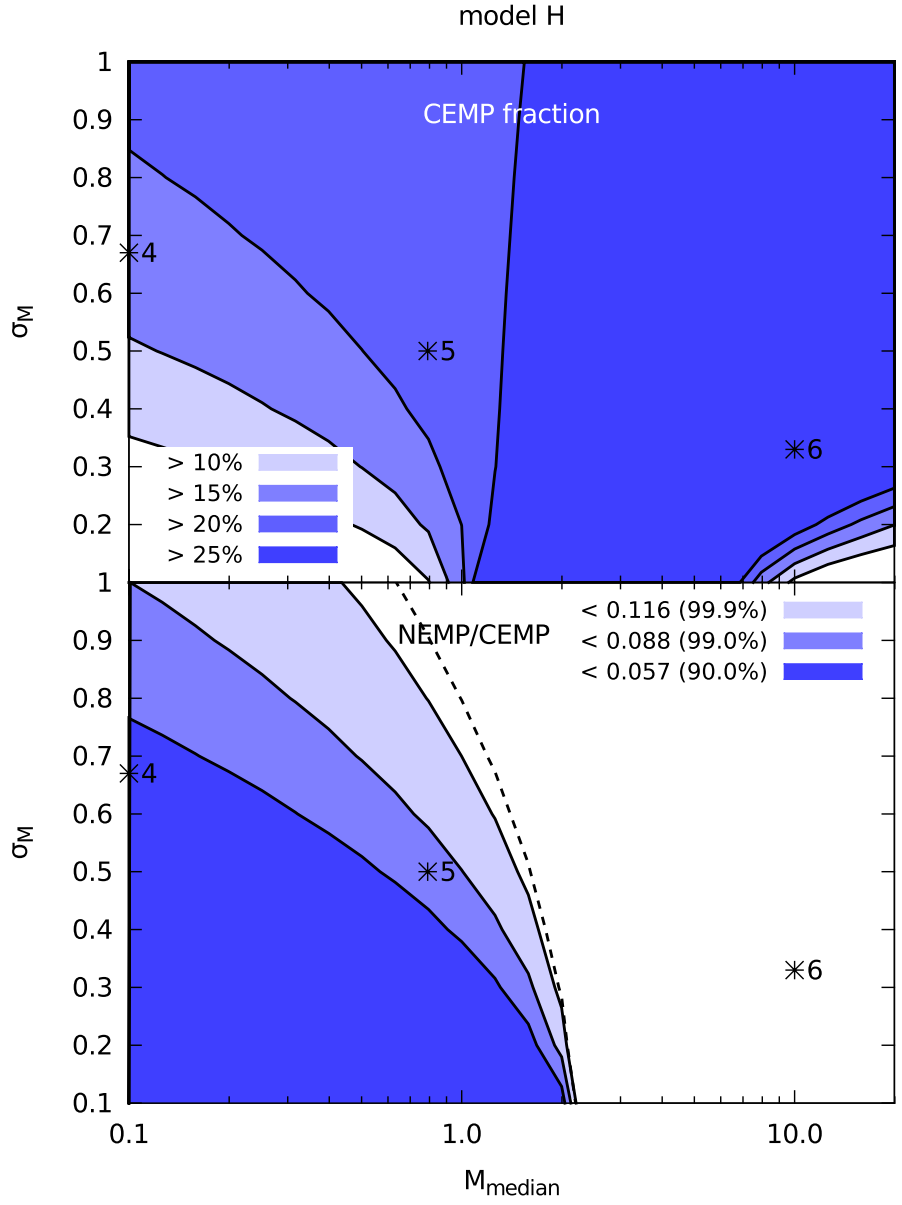

Fig. 5. Same as Fig. 3 for model H, without thermohaline mixing.

analysis of Komiya et al. (2007), the main difference being that we use our detailed population synthesis models as input and that we also consider the NEMP/CEMP fraction $f_{\mathrm{NEMP}}$ as a constraint.

Figure 3 demonstrates that Model A can only reproduce the observed CEMP fraction for $M_{\text {med }}>1 M_{\odot}$ and small values of $\sigma_{M}$, i.e. only for very narrow, top-heavy forms of the IMF. On the other hand, the upper limit on $f_{\text {NEMP }}$ only allows small $M_{\text {med }}$ and again favours small values of $\sigma_{M}$. To reproduce both with our default model, we would require a highly fine-tuned IMF with $M_{\text {med }} \approx 2 M_{\odot}$ and $\sigma_{M} \lesssim 0.2$, i.e. one which only produces intermediate-mass stars in a small mass range.

These stringent constraints are relieved with Model G, which produces many more CEMP stars but essentially the same number of NEMP stars, and thus a lower $f_{\text {NEMP. In this case a wider }}$ range of IMFs with $M_{\text {med }}<2 M_{\odot}$ is able to reproduce $f_{\mathrm{NEMP}}$, although a large CEMP fraction $(>15 \%)$ still requires a rather top-heavy IMF. In this case, however, CEMP fractions $>10 \%$ are also produced by a variety of broad, bottom-heavy IMFs. There is a band of values of $M_{\text {med }}$ and $\sigma_{M}$, which includes the Miller \& Scalo and Lucatello IMFs, that allows CEMP fractions $>10 \%$ with acceptable $f_{\text {NEMP }}$ values. In Model $\mathrm{H}$ both the CEMP and NEMP fractions increase with respect to Model G, while $f_{\text {NEMP }}$ remains almost unchanged. This model allows a range of bottom-heavy IMFs $\left(M_{\text {med }}<2 M_{\odot}\right)$ that reproduce both $f_{\mathrm{NEMP}}$ and a CEMP fraction of at least $15 \%$. If we consider only CNEMP stars (dashed lines in Figs. 3-5) the ranges of allowed IMF shapes do not change substantially, except that 
for model G a range of broad IMFs with $M_{\text {med }}>2 M_{\odot}$ cannot be firmly excluded.

We conclude that only bottom-heavy IMFs are potentially able to reproduce both the observed CEMP fraction and the NEMP/CEMP fraction among stars with $-2.8 \leq[\mathrm{Fe} / \mathrm{H}] \leq-1.8$, provided that efficient dredge-up of carbon occurs in low-mass AGB stars. On the other hand, top-heavy IMFs (with $M_{\text {med }}>$ $2 M_{\odot}$ ) are unable to produce a low enough $f_{\mathrm{NEMP}}$, if indeed HBB occurs in metal-poor AGB stars with $M>2.7 M_{\odot}$ as detailed models indicate.

\section{Discussion}

In the following subsections we discuss how various uncertainties in our models and assumptions could affect our results.

\subsection{The lower mass limit for hot bottom burning}

The likelihood of NEMP star formation depends on which masses of star undergo hot bottom burning. The higher the minimum initial mass for the onset of HBB, the fewer NEMP stars will form. Our results are based on an adopted lower mass limit for $\mathrm{HBB}, M_{\mathrm{HBB}}$, of $2.7 M_{\odot}$ in accordance with the results of Karakas \& Lattanzio (2007). On the other hand, Suda et al. (2011) argue that if $M_{\mathrm{HBB}}$ is larger than $5 M_{\odot}$ then the observed NEMP/CEMP ratio for $[\mathrm{Fe} / \mathrm{H}]<-2.5$ is compatible with the top-heavy IMF of Komiya et al. (2007).

The lower mass limit for HBB is thus an important quantity. Unfortunately, there is some uncertainty in this quantity, with different stellar evolution codes giving different values. At $Z=$ $10^{-4}$, Herwig (2004) finds $M_{\mathrm{HBB}} \approx 3.5 M_{\odot}$ for his models which include convective overshooting. A similar value is obtained by Stancliffe \& Glebbeek (2008), though they find some CN cycling occurring in a $3 M_{\odot}$ model. Karakas (2010) finds mild HBB to occur in a $3 M_{\odot}$ model at this metallicity. At a higher metallicity of $Z=10^{-3}$, Weiss \& Ferguson (2009) find hot bottom burning does not set in until the initial mass is $\geq 5 M_{\odot}$.

Several groups have made grids of AGB models over a range of masses and metallicities. It is instructive to compare their results, as this gives some indication of the uncertainties in the determination of $M_{\mathrm{HBB}}$. Campbell \& Lattanzio (2008) find that HBB occurs for masses equal to $2 M_{\odot}$ and above for all the metallicities they study: from $[\mathrm{Fe} / \mathrm{H}]=-3$ right down to the metal-free population. Lau et al. (2009) find $M_{\mathrm{HBB}} \geq 3 M_{\odot}$ for models without overshooting for metallicities from $[\mathrm{Fe} / \mathrm{H}]=$ -2.3 down to $[\mathrm{Fe} / \mathrm{H}]=-6.3$, the lowest metallicity in their grid. When they include convective overshooting during the preAGB evolution $M_{\mathrm{HBB}}$ drops to $2 M_{\odot}$. In stark contrast to this is the work of Suda \& Fujimoto (2010) who do not find HBB in any of their grid of models, which spans metallicities from $[\mathrm{Fe} / \mathrm{H}]=-2$ down to $[\mathrm{Fe} / \mathrm{H}]=-5$ (plus $\mathrm{Z}=0$ ) and masses from $0.8 M_{\odot}$ to $9 M_{\odot}$.

The origin of these differences is not easy to determine. Certainly one contributing factor is that different groups use different mixing lengths. This will change the efficiency of convection and hence the mass at which HBB sets in. It seems likely that the efficiency of third dredge-up (one of the perennial uncertainties of AGB modelling) also affects the occurrence of HBB, with more efficient TDU likely to cause the onset of HBB at lower masses. Another possibility is the treatment of molecular opacities. Marigo (2007) found that when a star reaches $\mathrm{C} / \mathrm{O}>1$ then hot bottom burning can be quenched in some cases. This would shift $M_{\mathrm{HBB}}$ to higher masses, although Marigo (2007) expects the effect to be small for $Z<10^{-3}$.

\subsection{Non-canonical evolution}

The preceding discussion has focused solely on canonical models of stellar evolution, that is to say, models that only transport material by convection. But it has long been thought that some extra mixing process may be at work in AGB stars, circulating material from the base of the convective envelope to the hydrogen burning shell. Whether this is necessary or not is an open question at higher metallicities (see Karakas et al. 2010 and Busso et al. 2010 for both sides of the argument), but at low metallicity it seems likely that some extra mixing process is at work. (Stancliffe et al. 2009; Masseron et al. 2010; Lucatello et al. 2011). The physical cause of this extra mixing is unknown. Stancliffe (2010) has shown that thermohaline mixing (as calibrated to match the abundance changes on the RGB) is unable to affect nitrogen production in low-metallicity AGB stars.

The effect that extra mixing would have on nitrogen production is difficult to assess while the nature of the mechanism remains unknown. From observations of CEMP stars it seems that only a weak circulation is required, as these stars still show carbon enhancements in excess of nitrogen enhancements, and few CEMP stars show ${ }^{12} \mathrm{C} /{ }^{13} \mathrm{C}$ ratios as low as the $\mathrm{CNO}$ cycle equilibrium value. So while extra mixing appears to be needed, it seems unlikely that it will significantly change the minimum mass for HBB.

At low metallicity, AGB models predict mixing episodes not seen at higher metallicity. The intershell convection zone is able to penetrate into the H-rich envelope, sucking protons down to high temperatures and leading to a large release of energy from hydrogen burning. These dual shell flash events (or proton ingestion episodes) can lead to the intershell convection zone splitting into two regions. One is driven by helium burning and the other by hydrogen burning. In the hydrogen-burning driven convection zone, the $\mathrm{CN}$ cycle takes place and potentially the nitrogen abundance can be elevated when the convective envelope penetrates into this region. Several groups have recently attempted to model this phase, including Campbell \& Lattanzio (2008), Lau et al. (2009), Iwamoto (2009), Cristallo et al. (2009) and Suda \& Fujimoto (2010). However, these models are all based on 1D mixing length theory which is almost certainly incorrect in these circumstances. What is really needed are hydrodynamical simulations of this phase of evolution. Recently, Stancliffe et al. (2011) performed such simulations. They found serious deficiencies in the way $1 \mathrm{D}$ evolution codes model the process of proton ingestion, noting that the transport of hydrogen is not diffusive in character, and that the velocities of the flows involved are significantly in excess of those given by mixing length theory. Crucially, they also found no evidence of the convective region splitting into two zones, despite significant energy generation from hydrogen burning.

While dual shell flash events may enhance the nitrogen abundance, it seems unlikely that they would produce nitrogen-rich AGB stars, as this would require a large degree of hydrogen ingestion together with fairly complete $\mathrm{CN}$ cycling. Whether this is the case will have to remain unknown until more detailed multi-dimensional calculations can be made. In summary, it seems improbable that non-canonical evolution will produce nitrogen-rich AGB stars over a greater mass range than currently predicted.

\subsection{Mass transfer efficiency}

In our models mass transfer is treated as a combination of Roche-lobe overflow (RLOF) and Bondi-Hoyle wind accretion 
(Bondi \& Hoyle 1944). The modelling of both processes has substantial shortcomings when applied to binaries containing AGB mass donors. Roche-lobe overflow is relevant in binary systems that are close enough for the AGB star to fill its Roche lobe, and in most cases leads to unstable mass transfer and a common envelope. This evolution channel produces only a small fraction of the CEMP and NEMP stars in our models. However, the classical Roche geometry that we have assumed may not apply to binaries containing luminous AGB stars, where radiation pressure may strongly modify the sizes and shapes of the Roche lobes (Schuerman 1972; Dermine et al. 2009). This could affect the stability of RLOF and the number of CEMP stars and NEMP stars produced by the RLOF channel. Because AGB stars undergoing HBB are intrinsically brighter than lower-mass AGB stars, the effect may be largest on the formation of NEMP stars.

The dominant formation mechanism of CEMP and NEMP stars in our models is wind accretion. By adopting Bondi-Hoyle accretion we make the implicit assumption that the wind velocity exceeds the orbital velocity. This assumption breaks down when the mass-losing star is an AGB star in a relatively close orbit. AGB mass loss is driven by a combination of stellar pulsation and radiation pressure on dust. The wind is accelerated in the region where dust condenses, at a distance of several stellar radii. Hydrodynamical simulations show that when the companion orbits at a distance where the AGB wind has not yet been accelerated to its terminal velocity, the gas flow geometry resembles Roche-lobe overflow rather than a Bondi-Hoyle flow (Nagae et al. 2004; de Val-Borro et al. 2009; Mohamed \& Podsiadlowski 2007, 2011). In this transition regime of "wind Roche-lobe overflow" Mohamed (2010) and Mohamed \& Podsiadlowski (2011) find that the companion can accrete up to $50 \%$ of the mass lost by an AGB star, a much higher accretion efficiency than expected from the Bondi-Hoyle mechanism $(\$ 10 \%)$. Abate et al. (2012) have applied a simple model for wind-RLOF in a population synthesis study and find that this mechanism increases the occurrence of CEMP stars by a factor of up to 1.5 compared to Bondi-Hoyle accretion.

Of particular relevance for this study is the question whether a more realistic treatment of mass transfer would change the NEMP/CEMP ratio, i.e. whether it would affect the formation of NEMP stars in a different way than that of CEMP stars. This question is difficult to answer without dedicated mass transfer calculations for these cases. Such calculation are difficult to perform because mass loss of low-metallicity AGB stars is poorly understood and is not constrained by observations. If radiation pressure on dust plays a similar role as at solar metallicity, then one might expect a difference between massive AGB stars that undergo HBB and lower-mass AGB stars that do not. Low-mass AGB stars produce carbon-rich dust, which condenses easily at relatively high temperature and thus close to the star. On the other hand, stars that have undergone $\mathrm{HBB}$ eject gas that is nitrogen-rich and poor in $\mathrm{C}$ and $\mathrm{O}$, which may form dust much less efficiently and at much larger distance to the star, although little is known about dust formation under such circumstances ${ }^{4}$. Massive AGB stars may therefore accelerate their winds at larger distance than their lower-mass counterparts. In the framework of the wind-RLOF model, one may thus expect a larger increase in the number of NEMP stars than in the CEMP number. If this presumption is correct, the observed NEMP/CEMP ratio would constrain any modifications to the IMF even more strongly than we find in Sect. 4.

\footnotetext{
${ }^{4}$ An interesting test case is provided by $\eta$ Carinae (Gull 2008).
}

\subsection{The binary fraction}

The results we present are based on an assumed binary fraction of $100 \%$, i.e. we assume that the progenitor population of CEMP and NEMP stars consists only of binaries. For metalpoor halo stars very little is known with certainty about the multiplicity fraction, but the predominance of binaries among the CEMP-s stars suggests that binaries are as common in the halo as they are among Population I stars in the Galactic disk. It is thus reasonable to base our discussion on the observed multiplicity in Galactic disk populations, which is much better determined. Among nearby solar-type stars, Duquennoy \& Mayor (1991) found a multiplicity fraction of about $57 \%$ for solar-type stars, which was revised to $46 \pm 2 \%$ in a more recent analysis of a much larger sample by Raghavan et al. (2010). These studies concern stars of somewhat lower mass than the progenitors of CEMP stars. Perhaps more relevant, certainly for NEMP stars, is the binary fraction among intermediate-mass stars which was studied by Kouwenhoven et al. (2007) in the young OB association Sco OB2. They found a binary fraction of at least $70 \%$ (3- $\sigma$ lower limit) and probably much closer to $100 \%$. A similarly high overall binary fraction is found among massive O-type stars (Mason et al. 2009; Sana et al. 2012). A comparison of these results suggests that the binary fraction among Galactic disk stars is an increasing function of stellar mass, a conclusion also reached by Lada (2006) for low-mass stars. If a similar trend exists for metal-poor halo stars, then the predicted NEMP/CEMP number ratio from our models would be higher. This would impose an even stronger constraint on the IMF.

\section{Conclusions}

Our results show that the occurrence of NEMP stars, in particular the observed ratio of NEMP to CEMP stars, sets important constraints on the initial mass function of the early Galactic halo. In the mass-transfer scenario, the production of nitrogen by hot bottom burning in intermediate-mass AGB stars implies that any shift in the IMF towards more massive stars is accompanied by an increase in the NEMP/CEMP ratio. From the currently known census of 12 candidate NEMP stars with $\log g \leq 4$ we derive an upper limit to the NEMP/CEMP ratio of 0.15 (at $99 \%$ confidence). In the metallicity range $-2.8 \leq[\mathrm{Fe} / \mathrm{H}] \leq$ -1.8 only two candidate NEMP stars are known, and we find NEMP/CEMP $<0.09$ with $99 \%$ confidence. Our detailed AGB models at $[\mathrm{Fe} / \mathrm{H}]=-2.3$, in which $\mathrm{HBB}$ occurs in AGB stars with $M>2.7 M_{\odot}$, exclude IMFs with median masses of $\gtrsim 2 M_{\odot}$ for stars formed in this metallicity range. On the other hand the observed NEMP/CEMP ratio is quite compatible with the solarneighbourhood IMF, provided that low-metallicity AGB stars in the mass range $0.85-1.2 M_{\odot}$ are capable of dredging up carbon and turning a low-mass binary companion into a CEMP star, as we concluded in Paper I.

The sample of known NEMP stars mostly have $[\mathrm{Fe} / \mathrm{H}]<$ -2.8 , which suggests that the NEMP/CEMP ratio is a strong function of metallicity. At $[\mathrm{Fe} / \mathrm{H}]<-2.8$ the observed NEMP/ CEMP ratio is approximately 0.27 (with a $99 \%$ confidence upper limit of 0.51 ). From currently available model sets of AGB stars it is not clear how the mass limits for HBB behave at such low metallicities, and therefore it remains unclear exactly what the implications are for the IMF among the most metalpoor halo stars. Assuming HBB occurs for similar masses as at $[\mathrm{Fe} / \mathrm{H}]=-2.3$, the observed NEMP/CEMP ratio allows and possibly requires - a shift in the IMF towards intermediatemass stars. Nevertheless, a top-heavy IMF such as suggested by 
Komiya et al. (2007) remains firmly excluded, unless HBB essentially shuts off at the lowest metallicities (Suda et al. 2011). A larger census of NEMP stars would be very valuable to put firmer constraints on possible changes to the IMF at low metallicity.

Acknowledgements. We thank the anonymous referee for valuable comments that helped us improve the paper. R.G.I. was funded by a Marie-Curie fellowship while in Brussels. The research leading to these results has received funding from the Seventh Framework Programme of the European Community under grant agreement 220440. R.J.S. is a Stromlo fellow. During his time at Monash University, he was funded through the Australian Research Council Discovery Projects scheme, under grant DP0879472. E.G. acknowledges support by a NWO VENI fellowship in Nijmegen.

\section{References}

Abate, C., Pols, O. R., Izzard, R. G., Mohamed, S. S., \& de Mink, S. E. 2012, A\&A, submitted

Abia, C., Domínguez, I., Straniero, O., et al. 2001, ApJ, 557, 126

Andrievsky, S. M., Spite, M., Korotin, S. A., et al. 2007, A\&A, 464, 1081

Andrievsky, S. M., Spite, M., Korotin, S. A., et al. 2010, A\&A, 509, A88

Andrievsky, S. M., Spite, F., Korotin, S. A., et al. 2011, A\&A, 530, A105

Aoki, W., Norris, J. E., Ryan, S. G., Beers, T. C., \& Ando, H. 2002, ApJ, 567, 1166

Aoki, W., Frebel, A., Christlieb, N., et al. 2006, ApJ, 639, 897

Aoki, W., Beers, T. C., Christlieb, N., et al. 2007, ApJ, 655, 492

Aoki, W., Beers, T. C., Sivarani, T., et al. 2008, ApJ, 678, 1351

Barklem, P. S., Christlieb, N., Beers, T. C., et al. 2005, A\&A, 439, 129

Beers, T. C., \& Christlieb, N. 2005, ARA\&A, 43, 531

Bondi, H., \& Hoyle, F. 1944, MNRAS, 104, 273

Busso, M., Palmerini, S., Maiorca, E., et al. 2010, ApJ, 717, L47

Campbell, S. W., \& Lattanzio, J. C. 2008, in First Stars III, AIP Conf. Ser., 990, 315

Carollo, D., Beers, T. C., Bovy, J., et al. 2012, ApJ, 744, 195

Cayrel, R., Depagne, E., Spite, M., et al. 2004, A\&A, 416, 1117

Cohen, J. G., McWilliam, A., Shectman, S., et al. 2006, AJ, 132, 137

Cohen, J. G., Christlieb, N., McWilliam, A., et al. 2008, ApJ, 672, 320

Cristallo, S., Piersanti, L., Straniero, O., et al. 2009, PASA, 26, 139

de Val-Borro, M., Karovska, M., \& Sasselov, D. 2009, ApJ, 700, 1148

Depagne, E., Hill, V., Spite, M., et al. 2002, A\&A, 390, 187

Dermine, T., Jorissen, A., Siess, L., \& Frankowski, A. 2009, A\&A, 507, 891

Duquennoy, A., \& Mayor, M. 1991, A\&A, 248, 485

Frebel, A., Christlieb, N., Norris, J. E., et al. 2006, ApJ, 652, 1585

Fulbright, J. P. 2000, AJ, 120, 1841

Gratton, R. G., Sneden, C., Carretta, E., \& Bragaglia, A. 2000, A\&A, 354, 169

Gratton, R. G., Carretta, E., Claudi, R., Lucatello, S., \& Barbieri, M. 2003, A\&A, 404, 187

Grevesse, N., Noels, A., \& Sauval, A. J. 1996, in Cosmic Abundances, eds. S. S. Holt, \& G. Sonneborn, ASP Conf. Ser., 99, 117

Gull, T. R. 2008, in Mass Loss from Stars and the Evolution of Stellar Clusters, eds. A. de Koter, L. J. Smith, \& L. B. F. M. Waters, ASP Conf. Ser., 388, 167 Herwig, F. 2004, ApJS, 155, 651

Iwamoto, N. 2009, PASA, 26, 145

Izzard, R. G., Tout, C. A., Karakas, A. I., \& Pols, O. R. 2004, MNRAS, 350, 407
Izzard, R. G., Dray, L. M., Karakas, A. I., Lugaro, M., \& Tout, C. A. 2006, A\&A, 460,565

Izzard, R. G., Lugaro, M., Karakas, A. I., Iliadis, C., \& van Raai, M. 2007, A\&A, 466, 641

Izzard, R. G., Glebbeek, E., Stancliffe, R. J., \& Pols, O. R. 2009, A\&A, 508, 1359

Johnson, J. A., Herwig, F., Beers, T. C., \& Christlieb, N. 2007, ApJ, 658, 1203

Karakas, A. I. 2010, MNRAS, 403, 1413

Karakas, A., \& Lattanzio, J. C. 2007, PASA, 24, 103

Karakas, A. I., Lattanzio, J. C., \& Pols, O. R. 2002, PASA, 19, 515

Karakas, A. I., Campbell, S. W., \& Stancliffe, R. J. 2010, ApJ, 713, 374

Kinman, T. D., Aoki, W., Beers, T. C., \& Brown, W. R. 2012, ApJ, 755, L18

Komiya, Y., Suda, T., Minaguchi, H., et al. 2007, ApJ, 658, 367

Kouwenhoven, M. B. N., Brown, A. G. A., Portegies Zwart, S. F., \& Kaper, L. 2007, A\&A, 474, 77

Kroupa, P., Tout, C. A., \& Gilmore, G. 1993, MNRAS, 262, 545

Lada, C. J. 2006, ApJ, 640, L63

Lau, H. H. B., Stancliffe, R. J., \& Tout, C. A. 2009, MNRAS, 396, 1046

Lucatello, S., Gratton, R. G., Beers, T. C., \& Carretta, E. 2005a, ApJ, 625, 833

Lucatello, S., Tsangarides, S., Beers, T. C., et al. 2005b, ApJ, 625, 825

Lucatello, S., Beers, T. C., Christlieb, N., et al. 2006, ApJ, 652, L37

Lucatello, S., Masseron, T., Johnson, J. A., Pignatari, M., \& Herwig, F. 2011, ApJ, 729, 40

Lugaro, M., Karakas, A. I., Stancliffe, R. J., \& Rijs, C. 2012, ApJ, 747, 2

Marigo, P. 2007, A\&A, 467, 1139

Mason, B. D., Hartkopf, W. I., Gies, D. R., Henry, T. J., \& Helsel, J. W. 2009, AJ, 137, 3358

Masseron, T., van Eck, S., Famaey, B., et al. 2006, A\&A, 455, 1059

Masseron, T., Johnson, J. A., Plez, B., et al. 2010, A\&A, 509, A93

Meynet, G., Hirschi, R., Ekstrom, S., et al. 2010, A\&A, 521, A30

Miller, G. E., \& Scalo, J. M. 1979, ApJS, 41, 513

Mohamed, S. 2010, Ph.D. Thesis, Oxford University, UK

Mohamed, S., \& Podsiadlowski, P. 2007, in 15th European Workshop on White Dwarfs, eds. R. Napiwotzki, \& M. R. Burleigh, ASP Conf. Ser., 372, 397

Mohamed, S., \& Podsiadlowski, P. 2011, in Why Galaxies Care about AGB Stars

II, eds. F. Kerschbaum, T. Lebzelter, \& R. F. Wing, ASP Conf. Ser., 445, 355

Nagae, T., Oka, K., Matsuda, T., et al. 2004, A\&A, 419, 335

Norris, J. E., Ryan, S. G., \& Beers, T. C. 2001, ApJ, 561, 1034

Raghavan, D., McAlister, H. A., Henry, T. J., et al. 2010, ApJS, 190, 1

Sana, H., de Mink, S. E., de Koter, A., et al. 2012, Science, 337, 444

Schuerman, D. W. 1972, Ap\&SS, 19, 351

Sivarani, T., Beers, T. C., Bonifacio, P., et al. 2006, A\&A, 459, 125

Spite, M., Cayrel, R., Plez, B., et al. 2005, A\&A, 430, 655

Spite, M., Cayrel, R., Hill, V., et al. 2006, A\&A, 455, 291

Stancliffe, R. J. 2009, MNRAS, 394, 1051

Stancliffe, R. J. 2010, MNRAS, 403, 505

Stancliffe, R. J., \& Glebbeek, E. 2008, MNRAS, 389, 1828

Stancliffe, R. J., Glebbeek, E., Izzard, R. G., \& Pols, O. R. 2007, A\&A, 464, L57

Stancliffe, R. J., Church, R. P., Angelou, G. C., \& Lattanzio, J. C. 2009, MNRAS, 396,2313

Stancliffe, R. J., Dearborn, D. S. P., Lattanzio, J. C., Heap, S. A., \& Campbell, S. W. 2011, ApJ, 742, 121

Suda, T., \& Fujimoto, M. Y. 2010, MNRAS, 405, 177

Suda, T., Katsuta, Y., Yamada, S., et al. 2008, PASJ, 60, 1159

Suda, T., Yamada, S., Katsuta, Y., et al. 2011, MNRAS, 412, 843

Weiss, A., \& Ferguson, J. W. 2009, A\&A, 508, 1343

Pages 12 to 13 are available in the electronic edition of the journal at http://www . aanda. org 


\section{Appendix A: Comments on individual NEMP stars}

In this section we comment on the reported observations for our potential NEMP stars. If these stars are truly polluted with material from hot-bottom burning companions they are expected to be rich in nitrogen, sodium and magnesium (e.g. Stancliffe 2009). Further processing of $\mathrm{C}$ and $\mathrm{N}$ because of extra mixing may occur in the star if is evolved beyond first dredge up.

Alpha elements such as calcium and titanium are enhanced by about $0.3-0.4 \mathrm{dex}$ in normal Galactic halo stars. Some lithium enhancement is also expected because of hot-bottom burning although there are many other processes through which it may be made (Iwamoto 2009; Stancliffe 2010). Heavy sprocess elements such as barium and lead are not thought to be produced in intermediate-mass AGB stars so are not expected to be significantly enhanced in NEMP stars (Lugaro et al. 2012).

The typical 1- $\sigma$ errors on abundance measurements $[\mathrm{X} / \mathrm{Fe}]$ are $0.2-0.3$ dex.

\section{A.1. CS22949-037}

The abundances of carbon and nitrogen in CS22949-037 are reported by Norris et al. (2001), Depagne et al. (2002), Cayrel et al. (2004) and Cohen et al. (2008) who all find [N/Fe] $>1$, $[\mathrm{C} / \mathrm{Fe}]>1$ and $[\mathrm{N} / \mathrm{C}]>0.5$. CS22949-037 is thus a CEMP and NEMP star. Spite et al. (2006) find ${ }^{12} \mathrm{C} /{ }^{13} \mathrm{C}=4$ which implies that the $\mathrm{CN}$ cycle is in equilibrium. These abundances are consistent with an intermediate mass AGB star undergoing hot bottom burning. The gravity of this star, $\log g=1.5-1.7$, implies that it has passed through first dredge-up and is at the point where extra mixing begins but has not yet had time to significantly alter surface abundances of carbon and nitrogen (see Figs. 2, 6, and 7 in Stancliffe et al. 2009).

Enhancements of sodium $([\mathrm{Na} / \mathrm{Fe}]=+1.57$, Andrievsky et al. 2007) and magnesium $([\mathrm{Mg} / \mathrm{Fe}]=+1.55$, Andrievsky et al. 2010) also suggest hot bottom burning is active. Barium is deficient $([\mathrm{Ba} / \mathrm{Fe}]=-0.84$, Norris et al. $2001 ;-0.66$, Cohen et al. 2008; -0.5, Andrievsky et al. 2011) and strontium is mildly enhanced $([\mathrm{Sr} / \mathrm{Fe}]=+0.18$, Cohen et al. $2008 ;+0.17$, Andrievsky et al. 2011). These abundances fit the canonical view that most s-process enhancement occurs in low-mass AGB stars.

\section{A.2. CS22960-053}

This star is enriched in carbon and nitrogen with $[\mathrm{C} / \mathrm{Fe}]=+2.05$ and $[\mathrm{N} / \mathrm{Fe}]=+3.06$ according to Aoki et al. (2007) but in contrast Johnson et al. (2007) find $[\mathrm{C} / \mathrm{Fe}]=[\mathrm{N} / \mathrm{Fe}]=+1.15$. The discrepancy in nitrogen abundances may well be due to the respective use of $\mathrm{CN}$ and $\mathrm{NH}$ as abundance indicators. We can only conclude that this system may be a NEMP star. The star is also enhanced in magnesium $([\mathrm{Mg} / \mathrm{Fe}]=0.65)$ and barium $([\mathrm{Ba} / \mathrm{Fe}]=0.86)$.

\section{A.3. CS29528-041}

If we assume that the molecular abundance measurements of Sivarani et al. (2006), $[\mathrm{CH} / \mathrm{Fe}]=1.59,[\mathrm{CN} / \mathrm{Fe}]=3.07$ and $[\mathrm{NH} / \mathrm{Fe}]=3.00$, are indicative of elemental abundances then this star satisfies our (C)NEMP criteria. With $\log g=4.0$ it is unevolved and there is no mixing on the RGB. Sodium is enhanced, $[\mathrm{Na} / \mathrm{Fe}]=1.20$, but magnesium is not, $[\mathrm{Mg} / \mathrm{Fe}]=0.40$, especially when compared to other alpha elements such as calcium and titanium which show similar enhancements. CS29528-041 is rich in lithium, $\log \epsilon_{\mathrm{Li}}=1.71$, and barium $[\mathrm{Ba} / \mathrm{Fe}]=0.97$.

\section{A.4. CS30314-067}

CS30314-067 classifies as a NEMP star according to the abundances determined by Aoki et al. (2002), $[\mathrm{C} / \mathrm{Fe}]=+0.5$ and $[\mathrm{N} / \mathrm{Fe}]=+1.20$, but Johnson et al. (2007) instead find $[\mathrm{C} / \mathrm{Fe}]=$ +0.25 and $[\mathrm{N} / \mathrm{Fe}]=+0.50$. Some of the discrepancy may result from the different nitrogen abundance indicators used and the different adopted gravities, although the Aoki et al. (2002) study also has greater resolution.

\section{A.5. CS30322-023}

Masseron et al. (2006) have suggested that CS30322-023 is an AGB star because its gravity is low, $\log g=-0.3$, but this is contradicted by Aoki et al. (2007) who claim $\log g=1.0$. In either case extra mixing on the RGB may have occurred. Both Masseron et al. (2006) and Aoki et al. (2007) claim [C/Fe] = +0.6 and significant nitrogen enrichment $([\mathrm{N} / \mathrm{Fe}]=+2.81$ and +2.47 respectively) which suggest this is a NEMP star. Its abundances of sodium, magnesium and heavy elements are also enhanced $([\mathrm{Ba} / \mathrm{Fe}] \approx+0.6$ and $[\mathrm{Pb} / \mathrm{Fe}]=+1.49)$.

\section{A.6. HD 25329}

HD 25329 is an unevolved star $(\log g=4.6)$ with $[\mathrm{C} / \mathrm{Fe}]=$ $0.1,[\mathrm{~N} / \mathrm{Fe}]=+1.0$ and ${ }^{12} \mathrm{C} /{ }^{13} \mathrm{C}>40$ (Fulbright 2000, Gratton et al. 2000 and Gratton et al. 2003). Its only slight enhancements in sodium and magnesium $([\mathrm{Na} / \mathrm{Fe}]=+0.24$ and $[\mathrm{Mg} / \mathrm{Fe}]=$ +0.59 ; Gratton et al. 2003) suggest it has not been polluted by a hot bottom burning AGB star. The origin of nitrogen in this object remains unexplained.

\section{A.7. HD 206983}

The metallicity of HD 206983 is rather large, $[\mathrm{Fe} / \mathrm{H}]=-0.99$ (Masseron et al. 2010), suggesting it is a nitrogen-rich $\mathrm{CH}$-star rather than a NEMP star. This is supported by an enhancement of barium, $[\mathrm{Ba} / \mathrm{Fe}]=+0.92$ (Masseron et al. 2010).

\section{A.8. HE0400-2030}

The carbon- and nitrogen-rich star HE0400-2030, with [C/Fe] = +1.14 and $[\mathrm{N} / \mathrm{Fe}]=+2.75$ according to high-resolution observations $(R=50,000)$ of Aoki et al. (2007), qualifies as a CNEMP star. Lower-resolution observations $(R=20,000)$ of Lucatello et al. (2006) find less enhancement, $[\mathrm{N} / \mathrm{Fe}]=+1.0$ and $[\mathrm{C} / \mathrm{Fe}]=+0.8$. Sodium $([\mathrm{Na} / \mathrm{Fe}]=+0.71)$, magnesium $([\mathrm{Mg} / \mathrm{Fe}]=+0.62)$ and barium $([\mathrm{Ba} / \mathrm{Fe}]=+1.64)$ are also enhanced.

If this star accreted from a hot bottom burning companion, then the accreted material must have been diluted during the main sequence (e.g. by thermohaline mixing, Stancliffe et al. 2007), as the sodium and magnesium abundances are lower than predicted by theoretical models.

\section{A.9. HE1031-0020}

Cohen et al. (2006) find $[\mathrm{C} / \mathrm{Fe}]=+1.63$ and $[\mathrm{N} / \mathrm{Fe}]=+2.48$ for HE1031-002, i.e. it is a CNEMP star. With $\log g=2.2$ it is a moderately evolved giant and should have finished first dredge up without significant further extra mixing. Magnesium is slightly enhanced $[\mathrm{Mg} / \mathrm{Fe}]=+0.5$ but sodium is not measured. Calcium, heavy s-process elements and lead are enhanced, 
$[\mathrm{Ca} / \mathrm{Fe}]=+1.12$, $[\mathrm{hs} / \mathrm{ls}]>+1$ and $[\mathrm{Pb} / \mathrm{Fe}]=+2.66$, suggest an odd evolutionary history for this star which does not necessarily involve a hot bottom burning companion.

\section{A.10. $\mathrm{HE} 1337+0012$}

The most extensive set of abundance measurements for the NEMP star HE1337+0012 (also known as G64-12) is provided by Aoki et al. (2006) who find $[\mathrm{C} / \mathrm{Fe}]=+0.49$ and $[\mathrm{N} / \mathrm{Fe}]=+1.42$. The sodium abundance is low, $[\mathrm{Na} / \mathrm{Fe}]=-1.1$, so HE1337+0012 probably did not accrete material from a hot bottom burning companion despite its NEMP status.

\section{A.11. HE1410+0213}

This star is in the samples of both Cohen et al. (2006) and Masseron et al. (2010) but the authors disagree on its surface gravity with $\log g=3.5$ and 2.0 respectively. This gives incompatible abundances of $[\mathrm{C} / \mathrm{Fe}]=+2.33$ and $[\mathrm{N} / \mathrm{Fe}]=+2.94$ according to Masseron et al. (2010) and $[\mathrm{C} / \mathrm{Fe}]=+1.83$ and $[\mathrm{N} / \mathrm{Fe}]=+1.73$ from Cohen et al. (2006). Given the similar resolution of the studies it is unclear which is correct.

\section{A.12. HE1413-1954}

Lucatello et al. (2006) find this is an unevolved $(\log g>4)$ CNEMP star with $[\mathrm{N} / \mathrm{Fe}]=+2.5$ and $[\mathrm{C} / \mathrm{Fe}]=+1.7$. Barklem et al. (2005) measure $\log g=4.59$, $[\mathrm{C} / \mathrm{Fe}]=+1.45$ and $[\mathrm{Sr} / \mathrm{Fe}]=-0.47$. The lack of further abundance measurements prevents a reliable determination of the origin of the nitrogen in this star.

\section{A.13. HE2150-0825 and HE2253-4217}

HE2150-0825 and HE2253-4217 are NEMP stars according to the carbon and nitrogen abundances measured by Lucatello et al. (2006) who find $[\mathrm{C} / \mathrm{Fe}]=+0.3$ and $[\mathrm{N} / \mathrm{Fe}]=+1.3$, and $[\mathrm{C} / \mathrm{Fe}]=+1.0$ and $[\mathrm{N} / \mathrm{Fe}]=+1.5$ respectively. The status of HE2253-4217 as a NEMP is marginal with $[\mathrm{N} / \mathrm{C}]=0.5$, while HE2150-0825 has a low total $\mathrm{CN}$ abundance, $[(\mathrm{C}+\mathrm{N}) / \mathrm{Fe}]=$ 0.76 .

\section{A.14. CS29528-028 and SDSS1707+58}

Neither CS29528-028 nor SDSS1707+58 has measured nitrogen, so they are not classed as NEMP stars, but their sodium and magnesium abundances are unusual. CS29528-028 is an unevolved star with $\log g=4.0$. Aoki et al. (2007) report that CS29528-028 has $[\mathrm{Na} / \mathrm{Fe}]=+2.68$ and $[\mathrm{Mg} / \mathrm{Fe}]=+1.69 \mathrm{de}-$ spite a normal calcium abundance $([\mathrm{Ca} / \mathrm{Fe}]=+0.46)$. Its barium abundance is $[\mathrm{Ba} / \mathrm{Fe}]=+3.27$.

SDSS1707 +58 is similarly enhanced with $[\mathrm{Na} / \mathrm{Fe}]=+2.71$ and $[\mathrm{Mg} / \mathrm{Fe}]=+1.13$ as well as $[\mathrm{Ba} / \mathrm{Fe}]=+3.40$ (Aoki et al. 2008). This star was recently found to be an RR Lyr star by Kinman et al. (2012), who also find a lower metallicity $[\mathrm{Fe} / \mathrm{H}]=-2.92$ and somewhat lower sodium and barium abundances.

Based on their sodium and magnesium abundances, these stars are quite possibly polluted by a companion undergoing hot bottom burning, although their barium enhancements do not support this view. Unfortunately, until their nitrogen abundance and carbon isotopic ratios are measured the status of these stars remains unknown. 